\title{
On multi-valued maps with images in the space of closed subsets of a metric space
}

\author{
Evgeny S Zhukovskiy and Elena A Panasenko*
}

"Correspondence:

panlena_t@mail.ru

Institute of Mathematics, Physics, and Computer Sciences, Tambov

State University named after G.R.

Derzhavin, Tambov, Russia

\begin{abstract}
A new metric in the space $\operatorname{clos}(X)$ of all closed subsets of a metric space $X$ is proposed. This metric, unlike the generalized Hausdorff metric, takes finite values only, and the convergence of a sequence of closed sets $H^{i}, i=1,2, \ldots$, with respect to this metric is equivalent to the convergence (in the sense of Hausdorff) for any $r \geq 0$ of the unions of $H$ ' with a closed 'exterior ball' of radius $r$. Using this metric allows one to investigate multi-valued maps that have images in $\operatorname{clos}(X)$ and are not continuous in the Hausdorff metric. In the work, the necessary and sufficient conditions for a multi-valued map to be continuous and Lipschitz with respect to the metric presented are studied, a connection of these properties with their analogues in the Hausdorff metric is derived, and a generalization of the Nadler fixed point theorem is obtained.
\end{abstract}

MSC: 47H04;47H10;54E35

Keywords: metric space; multi-valued map; fixed point

\section{Introduction}

Let $\mathbb{N} \doteq\{1,2,3, \ldots\} ; \mathbb{Z} \doteq\{\ldots,-2,-1,0,1,2, \ldots\} ; \mathbb{R}_{+} \doteq[0, \infty)$. Given a metric space $\left(X, \varrho_{X}\right)$, we use the following notation: $\varrho(x, M) \doteq \inf _{y \in M} \varrho_{X}(x, y)$ is the distance in $X$ from a point $x$ to a set $M ; d(N, M) \doteq \sup _{x \in N} \varrho(x, M)$ is the deviation in the sense of Hausdorff of a set $N$ from the set $M ; S_{r}\left(x_{0}\right) \doteq\left\{x \in X: \varrho_{X}\left(x, x_{0}\right)=r\right\}, O_{r}\left(x_{0}\right) \doteq\left\{x \in X: \varrho_{X}\left(x, x_{0}\right) \leq r\right\}$, $O_{r}^{o}\left(x_{0}\right) \doteq\left\{x \in X: \varrho_{X}\left(x, x_{0}\right)<r\right\}$ are, respectively, a sphere, a closed and an open ball of radius $r>0$ centered at $x_{0}$ in the space $X ; S_{0}\left(x_{0}\right)=O_{0}\left(x_{0}\right)=\left\{x_{0}\right\} ; O_{0}^{o}\left(x_{0}\right)=\varnothing ; \overline{O_{r}^{o}\left(x_{0}\right)} \doteq$ $\left\{x \in X: \varrho_{X}\left(x, x_{0}\right) \geq r\right\}$ is a closed 'exterior ball', i.e., the compliment to an open ball; $O_{r}^{o}(M) \doteq \bigcup_{x_{0} \in M}\left\{x \in X: \varrho_{X}\left(x, x_{0}\right)<r\right\}$ is an $r$-neighborhood of $M \subset X$. By $\operatorname{clbd}(X)$ denote the space of all nonempty closed bounded subsets of $X$. This space we endow with the Hausdorff metric dist:

$$
\operatorname{dist}(A, B)=\max \{d(A, B) ; d(B, A)\}, \quad A, B \in \operatorname{clbd}(X) .
$$

In this article, multi-valued maps having images in the space $\operatorname{clos}(X)$ of all nonempty closed subsets of a given metric space $X$ are investigated. The necessity of studying such maps arises, for example, in the problems concerning asymptotic behavior of controlled systems trajectories $[1,2]$. Treating the space $\operatorname{clos}(X)$ in pair with the Hausdorff metric dist (called, in this case, the generalized Hausdorff metric, meaning it may equal infinity) does not lead to substantial results if, for instance, the corresponding maps have images

(c) 2013 Zhukovskiy and Panasenko; licensee Springer. This is an Open Access article distributed under the terms of the Creative Commons Attribution License (http://creativecommons.org/licenses/by/2.0), which permits unrestricted use, distribution, and reproduction in any medium, provided the original work is properly cited. 
that are infinitely distant from each other (such maps, obviously, are not continuous, and the classical methods of analysis are not applicable here). As shown in [1-3], a way to overcome this difficulty and make it possible to use the known methods and standard techniques is to construct in the space $\operatorname{clos}(X)$ a metric satisfying the following conditions:

(1) the distance between any closed sets is finite;

(2) if a sequence of closed sets is convergent with respect to the Hausdorff metric, then it is convergent with respect to the 'new' metric;

(3) the convergence of a sequence $\left\{H^{i}\right\}_{i=1}^{\infty} \subset \operatorname{clos}(X)$ means the convergence for any $r>0$ (with respect to the Hausdorff metric) of the sequence of bounded subsets $H_{r}^{i} \subset H^{i}, H_{r}^{i} \subset O_{r}$, such that $\bigcup_{i} H_{r}^{i}=H^{i}$ and $H_{r_{1}}^{i} \subset H_{r_{2}}^{i}$ as soon as $r_{1}<r_{2}$.

For the space of convex closed subsets of $\mathbb{R}^{n}$, in [2], a metric (called the HausdorffBebutov metric) satisfying (1)-(3) was suggested. It allowed to investigate differential inclusions with convex unbounded right-hand sides. For studying multi-valued maps with non-convex unbounded images in $\mathbb{R}^{n}$, the Hausdorff-Bebutov metric turns out to be inefficient since, considered in the space $\operatorname{clos}\left(\mathbb{R}^{n}\right)$, it does not meet requirements (2) and (3). Moreover, in the structure of the Hausdorff-Bebutov metric, the local compactness of $\mathbb{R}^{n}$ is fundamental, so the definition of this metric itself cannot be used for an arbitrary metric space $X$. In what follows, a different construction for a metric in the space $\operatorname{clos}(X)$ is offered. It fulfills all the listed requirements and can be used in the case of arbitrary $X$.

Fix a point $\theta \in X$ and denote $O_{r}^{o} \doteq O_{r}^{o}(\theta), O_{r} \doteq O_{r}(\theta), S_{r} \doteq S_{r}(\theta), \overline{O_{r}^{o}} \doteq \overline{O_{r}^{o}(\theta)}$. For every $r \geq 0$, define the operator $\mathfrak{S}_{r}: \operatorname{clos}(X) \rightarrow \operatorname{clos}(X)$ by the equality

$$
\mathfrak{S}_{r} H \doteq H \cup \overline{O_{r}^{o}} \text {. }
$$

Next, for any sets $F, G \in \operatorname{clos}(X)$, suppose

$$
\begin{aligned}
& \rho^{o}(F, G) \doteq|\varrho(\theta, F)-\varrho(\theta, G)|, \\
& \rho^{\mathfrak{S}}(F, G) \doteq \sup _{r>0} \min \left\{\operatorname{dist}\left(\mathfrak{S}_{r} F, \mathfrak{S}_{r} G\right), \frac{1}{r}\right\},
\end{aligned}
$$

and

$$
\rho_{\mathrm{cl}}(F, G) \doteq \rho^{o}(F, G)+\rho^{\mathfrak{S}}(F, G) .
$$

The function $\rho_{\mathrm{cl}}$ takes finite values only and, as it will be shown later, satisfies all the axioms of a metric. This metric first was introduced by the authors in [4] for the space clos $\left(\mathbb{R}^{n}\right)$ of all nonempty closed subsets of a finite dimensional space. In [5], the metric $\rho_{\mathrm{cl}}$ was used for studying the dynamical system of translations in the space of multi-valued maps with images in $\operatorname{clos}\left(\mathbb{R}^{n}\right)$.

In the work, we point out the benefits the metric $\rho_{\mathrm{cl}}$ gives when it comes to dealing with continuous and Lipschitz multi-valued maps. We emphasize that a crucial role here is played by the construction (2) used in the metric $\rho_{\mathrm{cl}}$, i.e., associating to every set $H \in$ $\operatorname{clos}(X)$ the set $\mathfrak{S}_{r} H \in \operatorname{clos}(X), r \geq 0$ (representing the union of $H$ with the closed 'exterior ball' of radius $r$ centered at $\theta$ ). ${ }^{\text {a }}$ For a map $F: X \rightarrow \operatorname{clos}(X)$, such an 'extension' of the values $F(x)$ allows, in particular, to obtain a fixed point theorem (which will be proved in the last section of the paper) for multi-valued maps that in the Hausdorff metric may 
not be contracting or even continuous and to which the known fixed point principles (for example, Nadler's theorem) are not applicable. One can also use this idea for a map $F$ : $X \rightarrow \operatorname{clbd}(X)$ that is not contracting or continuous. In many cases, after the corresponding extension, the map $\mathfrak{S}_{r} F: X \rightarrow \operatorname{clos}(X)$ gets these properties.

\section{The space $\left(\operatorname{clos}(X), \rho_{\mathrm{cl}}\right)$}

We start with studying the space $\left(\operatorname{clos}(X), \rho_{\mathrm{cl}}\right)$.

Fix a point $\theta \in X$ and set $R^{*} \doteq \sup _{x \in X} \varrho_{X}(\theta, x)$. We are interested, above all, in unbounded metric spaces, i.e., in the situation when $R^{*}=\infty$, but all the results obtained from now on hold also in the case of $R^{*}<\infty$. Set

$$
P \doteq \begin{cases}{\left[0, R^{*}\right],} & \text { if } R^{*}<\infty \text { and } \exists x \in X: \varrho_{X}(\theta, x)=R^{*} \\ {\left[0, R^{*}\right),} & \text { otherwise, }\end{cases}
$$

and define the function $D: P \times \mathbb{R}_{+} \rightarrow \mathbb{R}_{+}$in the following way:

$$
D(p, r) \doteq \begin{cases}0, & \text { if } r \leq p, \\ \operatorname{dist}\left(\overline{O_{p}^{o}}, \overline{O_{r}^{o}}\right), & \text { if } r>p, r \in P \\ \sup _{x, u \in \overline{O_{p}^{o}}} \varrho_{X}(x, u), & \text { if } r>p, r \notin P\end{cases}
$$

(the latter occurs only if $R^{*}<\infty$ ).

We will need the following properties of this function: for every $p \in P$, the function $D(p, \cdot): \mathbb{R}_{+} \rightarrow \mathbb{R}_{+}$is non-decreasing; for every $r \in \mathbb{R}_{+}$, the function $D(\cdot, r): P \rightarrow \mathbb{R}_{+}$is nonincreasing.

Show that $D(p, \cdot)$ is non-decreasing. Let $p<r_{1}<r_{2}$. If $r_{1}, r_{2} \in P$, then from $\overline{O_{r_{2}}^{o}} \subset \overline{O_{r_{1}}^{o}} \subset$ $\overline{O_{p}^{o}}$ it follows that $D(p, r)=\operatorname{dist}\left(\overline{O_{p}^{o}}, \overline{O_{r_{1}}^{o}}\right) \leq \operatorname{dist}\left(\overline{O_{p}^{o}}, \overline{O_{r_{2}}^{o}}\right)=D\left(p, r_{2}\right)$. In the case when $r_{1} \in P$, $r_{2} \notin P$ (this situation appears only for $R^{*}<\infty$ ), one gets

$$
D(p, r)=\operatorname{dist}\left(\overline{O_{p}^{o}}, \overline{O_{r_{1}}^{o}}\right) \leq \sup _{x \in \overline{O_{p}^{o}}, u \in \overline{O_{r_{1}}^{o}}} \varrho_{X}(x, u) \leq \sup _{x, u \in \overline{O_{p}^{o}}} \varrho_{X}(x, u)=D\left(p, r_{2}\right)
$$

Finally, if $r_{1}, r_{2} \notin P, R^{*}<\infty$, then $D\left(p, r_{1}\right)=D\left(p, r_{2}\right)$.

Let us illustrate the definition of the function $D$ on some concrete metric spaces.

Example 1 For any linear normed space $X$ with any choice of $\theta \in X$, the function $D: \mathbb{R}_{+}^{2} \rightarrow$ $\mathbb{R}_{+}$is given by $D(p, r)=r-p, r>p$.

If $X=\mathbb{Z}, \varrho_{\mathbb{Z}}(n, m)=|n-m| \forall n, m \in \mathbb{Z}$, then no matter what point is chosen as $\theta \in \mathbb{Z}$, for any $r, p \in \mathbb{R}_{+}, r>p$, the value of $D(p, r)$ is equal to the number of integers belonging to the interval $[p, r)$.

One gets the same values for $D: \mathbb{R}_{+}^{2} \rightarrow \mathbb{R}_{+}$if $X=\mathbb{N}, \varrho_{\mathbb{N}}(n, m)=|n-m| \forall n, m \in \mathbb{N}$, and $\theta=1$. If $\theta \neq 1$, the value $D(p, r)$ coincides with the number of integers in the set $[p, r)$ when $\theta-1 \geq r>p$ or $r>p>\theta-1$, and is equal to the number of integers in the set $[1, \theta+r)$ when $r>\theta-1 \geq p$.

Next, consider $X=[-1,2], \theta=0, \varrho_{X}(x, u)=|x-u|$. Then $R^{*}=2, P=[0,2]$, and for $r>p$, the function $D: P \times \mathbb{R}_{+} \rightarrow \mathbb{R}_{+}$is defined as follows: 
(1) if $0 \leq p \leq 1$, then

$$
D(p, r)= \begin{cases}r-p, & r \leq 1, \\ r+1, & 1<r \leq 2, \\ 3, & r>2\end{cases}
$$

(2) if $1 \leq p \leq 2$, then

$$
D(p, r)= \begin{cases}r-p, & r \leq 2, \\ 2-p, & r>2\end{cases}
$$

For arbitrary closed $F, G \subset X$ and $r \geq 0$, we determine now $\operatorname{dist}\left(\mathfrak{S}_{r} F, \mathfrak{S}_{r} G\right)$. Next, we show that this distance is finite and also give the properties of the function $\mathbb{R}_{+} \ni r \mapsto$ $\operatorname{dist}\left(\mathfrak{S}_{r} F, \mathfrak{S}_{r} G\right) \in \mathbb{R}_{+}$; these properties will be used in the sequel.

Lemma 1 Let $F, G \in \operatorname{clos}(X)$ be given. Then $\operatorname{dist}\left(\mathfrak{S}_{r} F, \mathfrak{S}_{r} G\right)<\infty$ for every $r \geq 0$ and the following statements hold true:

(1) the function $\mathbb{R}_{+} \ni r \mapsto \operatorname{dist}\left(\mathfrak{S}_{r} F, \mathfrak{S}_{r} G\right) \in \mathbb{R}_{+}$is non-decreasing;

(2) for every $r \geq 0$, the inequality

$$
\operatorname{dist}\left(\mathfrak{S}_{r} F, \mathfrak{S}_{r} G\right) \leq \operatorname{dist}(F, G)
$$

and the relation

$$
\lim _{r \rightarrow \infty} \operatorname{dist}\left(\mathfrak{S}_{r} F, \mathfrak{S}_{r} G\right)=\operatorname{dist}(F, G)
$$

hold;

(3) denoted $r * \min \{\varrho(\theta, F), \varrho(\theta, G)\}$, the inequality $\operatorname{dist}\left(\mathfrak{S}_{r} F, \mathfrak{S}_{r} G\right) \leq D(r * r)$ holds for each $r \geq 0$;

(4) denoted $r^{\prime \prime} \doteq \max \{\varrho(\theta, F), \varrho(\theta, G)\}$, the inequality $\rho^{o}(F, G) \leq \operatorname{dist}\left(\mathfrak{S}_{r} F, \mathfrak{S}_{r} G\right)$ holds for each $r \geq r^{*}$.

Proof First of all, note that for any sets $A, B, C \in \operatorname{clos}(X)$, one has

$$
\operatorname{dist}(A \cup C, B \cup C) \leq \operatorname{dist}(A, B) .
$$

To show this inequality, we estimate the deviation $d(A \cup C, B \cup C)$. Since

$$
\varrho(x, B \cup C) \leq \varrho(x, B) \leq d(A, B)
$$

for every $x \in A$ and

$$
\varrho(x, B \cup C)=0 \leq d(A, B)
$$

for every $x \in C$, we get the inequality $d(A \cup C, B \cup C) \leq d(A, B)$. Similarly, it can be checked that $d(B \cup C, A \cup C) \leq d(A, B)$. So, (8) is proved. 
From inequality (8), it follows that $\operatorname{dist}\left(\mathfrak{S}_{r} F, \mathfrak{S}_{r} G\right)<\infty$ for every $r \geq 0$. Indeed, this is obvious if $R^{*}<\infty$; in the case when $R^{*}=\infty$, one has $\mathfrak{S}_{r} F=\left(F \cap O_{r}\right) \cup \overline{O_{r}^{o}}, \mathfrak{S}_{r} G=\left(G \cap O_{r}\right) \cup$ $\overline{O_{r}^{o}}$ and hence,

$$
\operatorname{dist}\left(\mathfrak{S}_{r} F, \mathfrak{S}_{r} G\right) \leq \operatorname{dist}\left(F \cap O_{r}, G \cap O_{r}\right)<\infty .
$$

Using (8) one gets relation (6)

$$
\operatorname{dist}\left(\mathfrak{S}_{r} F, \mathfrak{S}_{r} G\right)=\operatorname{dist}\left(F \cup \overline{O_{r}^{o}}, G \cup \overline{O_{r}^{o}}\right) \leq \operatorname{dist}(F, G)
$$

Next, for $r_{2} \geq r_{1}, \mathfrak{S}_{r_{1}} F=\mathfrak{S}_{r_{2}} F \cup \overline{O_{r_{1}}^{o}}, \mathfrak{S}_{r_{1}} G=\mathfrak{S}_{r_{2}} G \cup \overline{O_{r_{1}}^{o}}$ hold. So, as a consequence of (8), the following holds:

$$
\operatorname{dist}\left(\mathfrak{S}_{r_{1}} F, \mathfrak{S}_{r_{1}} G\right) \leq \operatorname{dist}\left(\mathfrak{S}_{r_{2}} F, \mathfrak{S}_{r_{2}} G\right)
$$

Thus, the function $r \mapsto \operatorname{dist}\left(\mathfrak{S}_{r} F, \mathfrak{S}_{r} G\right)$ is non-decreasing.

Now, we prove equality (7). If $R^{*}<\infty$, then $\mathfrak{S}_{r} F=F, \mathfrak{S}_{r} G=G$ for $r>R^{*}$, so (7) is true.

Let $R^{*}=\infty$. First, suppose that $\operatorname{dist}(F, G)<\infty$. Since the function $r \mapsto \operatorname{dist}\left(\mathfrak{S}_{r} F, \mathfrak{S}_{r} G\right)$ is non-decreasing and bounded, there exists $\alpha \doteq \lim _{r \rightarrow \infty} \operatorname{dist}\left(\mathfrak{S}_{r} F, \mathfrak{S}_{r} G\right)$. Then for each $r$, the inequality $\alpha \geq \operatorname{dist}\left(\mathfrak{S}_{r} F, \mathfrak{S}_{r} G\right)$ takes place, and it follows that for any $\varepsilon>0$, every $x \in F$, and $r>\varrho_{X}(\theta, x)+\alpha+2 \varepsilon$, one has $x \in O_{\alpha+\varepsilon}^{o}\left(\mathfrak{S}_{r} G\right)$ and $x \notin \overline{O_{r-\varepsilon-\alpha}^{o}}$. Thus, taking into account $\overline{O_{r-\varepsilon-\alpha}^{o}} \supseteq O_{\alpha+\varepsilon}^{o}\left(\overline{O_{r}^{o}}\right)$, one gets $x \notin O_{\alpha+\varepsilon}^{o}\left(\overline{O_{r}^{o}}\right)$, i.e., $x \in O_{\alpha+\varepsilon}^{o}(G)$. As above, for each point $y \in G$, $y \in O_{\alpha+\varepsilon}^{o}(F)$ holds. Hence, $\operatorname{dist}(F, G) \leq \alpha+\varepsilon$ for any $\varepsilon>0$, which means that $\operatorname{dist}(F, G) \leq \alpha$. The inequality $\operatorname{dist}(F, G) \geq \alpha$ is easily obtained by passing to the limit in (6).

Now, let $\operatorname{dist}(F, G)=\infty$. If (7) is not true, then there exists $\alpha \doteq \lim _{r \rightarrow \infty} \operatorname{dist}\left(\mathfrak{S}_{r} F, \mathfrak{S}_{r} G\right)<$ $\infty$. So, arguing as before, one gets the inequality $\operatorname{dist}(F, G) \leq \alpha<\infty$, which contradicts the initial assumption. Thus, equality (7) is proved.

In order to prove statement (3), note that for every $r \leq r_{*}, \mathfrak{S}_{r} F=\mathfrak{S}_{r} G=\overline{O_{r}^{o}}$ holds and hence $\operatorname{dist}\left(\mathfrak{S}_{r} F, \mathfrak{S}_{r} G\right)=0$. If $r>r * r \in P$, then, according to the inclusions

$$
\mathfrak{S}_{r} F \subset \overline{O_{r}^{o}}, \quad \overline{O_{r}^{o}} \subset \mathfrak{S}_{r} G
$$

one gets

$$
d\left(\mathfrak{S}_{r} G, \mathfrak{S}_{r} F\right) \leq d\left(\overline{O_{r}^{o}}, \overline{O_{r *}^{o}}\right) \leq D\left(r_{*}, r\right)
$$

Similarly, it follows that $d\left(\mathfrak{S}_{r} F, \mathfrak{S}_{r} G\right) \leq D\left(r_{*}, r\right)$. So, $\operatorname{dist}\left(\mathfrak{S}_{r} F, \mathfrak{S}_{r} G\right) \leq D\left(r_{*}, r\right)$. For every $r \notin P, \mathfrak{S}_{r} G \subset \overline{O_{r o}^{o}}, \mathfrak{S}_{r} F \subset \overline{O_{r}^{o}}$ holds and therefore,

$$
\operatorname{dist}\left(\mathfrak{S}_{r} F, \mathfrak{S}_{r} G\right) \leq \sup _{x, u \in \overline{O_{r s}^{o}}} \varrho_{X}(x, u)=D\left(r_{*}, r\right)
$$

Property (4) is a consequence of the well-known estimate (see, e.g., [6])

$$
|\varrho(x, A)-\varrho(x, B)| \leq \operatorname{dist}(A, B), \quad \forall x \in X, \forall A, B \in \operatorname{clos}(X) .
$$


Indeed, assuming $A=\mathfrak{S}_{r} F, B=\mathfrak{S}_{r} G$ and taking into account that for every $r \geq r^{*}$, $\rho^{o}(F, G)=\rho^{o}\left(\mathfrak{S}_{r} F, \mathfrak{S}_{r} G\right)$ holds, one gets

$$
\rho^{o}(F, G)=\left|\varrho\left(\theta, \mathfrak{S}_{r} F\right)-\varrho\left(\theta, \mathfrak{S}_{r} G\right)\right| \leq \operatorname{dist}\left(\mathfrak{S}_{r} F, \mathfrak{S}_{r} G\right), \quad \forall r \geq r^{*}
$$

Theorem 1 Equality (5) defines a metric in the space clos $(X)$. If $X$ is a complete metric space, then $\left(\operatorname{clos}(X), \rho_{\mathrm{cl}}\right)$ is also complete.

Proof Show that $\rho_{\mathrm{cl}}$ satisfies all the axioms of a metric, i.e., that for any $F, G, H \in \operatorname{clos}(X)$, the following hold:

(1) $0 \leq \rho_{\mathrm{cl}}(F, G)<\infty$, and $\rho_{\mathrm{cl}}(F, G)=0$ if and only if $F=G$;

(2) $\rho_{\mathrm{cl}}(F, G)=\rho_{\mathrm{cl}}(G, F)$;

(3) $\rho_{\mathrm{cl}}(F, G) \leq \rho_{\mathrm{cl}}(F, H)+\rho_{\mathrm{cl}}(H, G)$.

It is obvious that $\rho_{\mathrm{cl}}(F, G) \geq 0$ and $\rho^{o}(F, G)<\infty$ for any $F, G \in \operatorname{clos}(X)$. According to Lemma 1 , one has

$$
\begin{aligned}
\rho^{\mathfrak{S}}(F, G) & \leq \sup _{r>0} \min \left\{D\left(r_{*}, r\right), \frac{1}{r}\right\} \leq \max \left\{\sup _{r \in[0,1]} D\left(r_{*}, r\right), \sup _{r>1} \frac{1}{r}\right\} \\
& =\max \left\{D\left(r_{*}, 1\right), 1\right\} \leq \max \{D(0,1), 1\} .
\end{aligned}
$$

Therefore, $\rho_{\mathrm{cl}}(F, G)<\infty$. Next,

$$
\begin{aligned}
\rho_{\mathrm{cl}}(F, G)=0 & \Leftrightarrow\left\{\begin{array}{l}
\rho^{o}(F, G)=0, \\
\operatorname{dist}\left(\mathfrak{S}_{r} F, \mathfrak{S}_{r} G\right)=0 \quad \forall r>0
\end{array}\right. \\
& \Leftrightarrow\left\{F \cap O_{r}^{o}=G \cap O_{r}^{o} \forall r>0\right\} \quad \Leftrightarrow \quad F=G .
\end{aligned}
$$

The symmetry of $\rho_{\mathrm{cl}}$ is straightforward; property (3) follows from the inequalities

$$
\begin{aligned}
& \rho^{o}(F, G) \leq \rho^{o}(F, H)+\rho^{o}(H, G), \\
& \operatorname{dist}\left(\mathfrak{S}_{r} F, \mathfrak{S}_{r} G\right) \leq \operatorname{dist}\left(\mathfrak{S}_{r} F, \mathfrak{S}_{r} H\right)+\operatorname{dist}\left(\mathfrak{S}_{r} H, \mathfrak{S}_{r} G\right) \quad \forall r \geq 0 .
\end{aligned}
$$

Thus, $\left(\operatorname{clos}(X), \rho_{\mathrm{cl}}\right)$ is a metric space.

Now, let $X$ be complete; show that $\left(\operatorname{clos}(X), \rho_{\mathrm{cl}}\right)$ is also a complete metric space.

Consider a fundamental sequence $\left\{F^{i}\right\}_{i=1}^{\infty} \subset \operatorname{clos}(X)$. For any $\varepsilon>0$, there is a number $N(\varepsilon)$ such that for all $i, j>N(\varepsilon)$, the following inequality takes place:

$$
\rho_{\mathrm{cl}}\left(F^{i}, F^{j}\right)=\left|\varrho\left(\theta, F^{i}\right)-\varrho\left(\theta, F^{j}\right)\right|+\sup _{r>0} \min \left\{\operatorname{dist}\left(\mathfrak{S}_{r} F^{i}, \mathfrak{S}_{r} F^{j}\right), \frac{1}{r}\right\} \leq \varepsilon .
$$

From (10), it follows that the number sequence $\left\{\varrho\left(\theta, F^{i}\right)\right\}_{i=1}^{\infty}$ is fundamental, so it is convergent; denote its limit by $r_{0}$; obviously, $r_{0} \geq 0$.

Take an arbitrary radius $r>r_{0}$ and consider the sequence $\left\{\mathfrak{S}_{r} F^{i}\right\}_{i=1}^{\infty} \subset \operatorname{clos}(X)$. It follows from (10) that for any $\varepsilon \in(0,1 / r)$ and all $i, j>N(\varepsilon)$, the inequality $\operatorname{dist}\left(\mathfrak{S}_{r} F^{i}, \mathfrak{S}_{r} F^{j}\right)<\varepsilon$ takes place. Show that for the sequence $\left\{\mathfrak{S}_{r} F^{i} \cap O_{r+1}\right\}_{i=1}^{\infty} \subset \operatorname{clbd}(X)$ and all $i, j>N(\varepsilon)$, the similar inequality holds:

$$
\operatorname{dist}\left(\mathfrak{S}_{r} F^{i} \cap O_{r+1}, \mathfrak{S}_{r} F^{j} \cap O_{r+1}\right)<\varepsilon .
$$


Without loss of generality, assume $\varepsilon<1$. Pick an arbitrary $x \in \mathfrak{S}_{r} F^{i} \cap O_{r+1}$. If $x \in O_{r+1} \backslash O_{r}^{o}$, then $\varrho\left(x, \mathfrak{S}_{r} F^{j} \cap O_{r+1}\right)=0$ since $O_{r+1} \backslash O_{r}^{o} \subset \mathfrak{S}_{r} F^{j} \cap O_{r+1}$. In the case when $x \in \mathfrak{S}_{r} F^{i} \cap O_{r}$, there exists a point $y \in \mathfrak{S}_{r} F^{j}$ satisfying the estimate $\varrho_{X}(x, y)<\varepsilon$. Held $y \in \overline{O_{r+1}^{o}}$, one gets $\varrho_{X}(x, y) \geq 1$, therefore $y \in \mathfrak{S}_{r} F^{j} \cap O_{r+1}$. So,

$$
d\left(\mathfrak{S}_{r} F^{i} \cap O_{r+1}, \mathfrak{S}_{r} F^{j} \cap O_{r+1}\right)<\varepsilon, \quad \forall i, j>N(\varepsilon) .
$$

Similarly, $d\left(\mathfrak{S}_{r} F^{j} \cap O_{r+1}, \mathfrak{S}_{r} F^{i} \cap O_{r+1}\right)<\varepsilon$ and relation (11) is proved.

The sequence $\left\{\mathfrak{S}_{r} F^{i} \cap O_{r+1}\right\}_{i=1}^{\infty} \subset \operatorname{clbd}(X)$ is fundamental with respect to the metric dist and since the space $(\operatorname{clbd}(X)$, dist) is complete (see [7]), this sequence converges to some 'limit' set $\mathfrak{F}_{r} \in \operatorname{clbd}(X)$. According to inequality (8), the following holds:

$$
\operatorname{dist}\left(\mathfrak{S}_{r} F^{i}, \mathfrak{S}_{r} \mathfrak{F}_{r}\right)=\operatorname{dist}\left(\left(\mathfrak{S}_{r} F^{i} \cap O_{r+1}\right) \cup \overline{O_{r}^{o}}, \mathfrak{F}_{r} \cup \overline{O_{r}^{o}}\right) \leq \operatorname{dist}\left(\mathfrak{S}_{r} F^{i} \cap O_{r+1}, \mathfrak{F}_{r}\right)
$$

and therefore $\mathfrak{S}_{r} F^{i} \rightarrow \mathfrak{S}_{r} \mathfrak{F}_{r}$. Moreover, $\varrho\left(\theta, \mathfrak{F}_{r}\right)=\varrho\left(\theta, \mathfrak{S}_{r} \mathfrak{F}_{r}\right)=r_{0}$ (this equality follows from statement (4) of Lemma 1).

For every $r>r_{0}$, the set $F_{r} \doteq \mathfrak{S}_{r} \mathfrak{F}_{r} \cap O_{r}^{o}$ is not empty for $\varrho\left(\theta, \mathfrak{S}_{r} \mathfrak{F}_{r}\right)=r_{0}$. Prove that for any $\bar{r}>r, \mathfrak{S}_{\bar{r}} \mathfrak{F}_{\bar{r}} \cap O_{r}^{o}=F_{r}$ holds. Let $x \in \mathfrak{S}_{\bar{r}} \mathfrak{F}_{\bar{r}} \cap O_{r}^{o}$. Then there exists a sequence $\left\{x_{i}\right\}_{i=1}^{\infty}, x_{i} \in$ $\mathfrak{S}_{\bar{r}} F^{i} \subset \mathfrak{S}_{r} F^{i}$, convergent to $x$. This means that $x \in \mathfrak{S}_{r} \mathfrak{F}_{r} ;$ moreover, $x \in O_{r}^{o}$. Conversely, let $x \in \mathfrak{S}_{r} \mathfrak{F}_{r} \cap O_{r}^{o}$, then $x \in \mathfrak{F}_{r}, x \notin \overline{O_{r}^{o}}$, and hence there is a sequence $\left\{x_{i}\right\}_{i=1}^{\infty}, x_{i} \in \mathfrak{S}_{r} F^{i}, x_{i} \rightarrow x$. Starting with some index $I$, all the members of this sequence satisfy the condition $x_{i} \notin \overline{O_{r}^{o}}$, and therefore $x_{i} \in F^{i} \subset \mathfrak{S}_{\bar{r}} F^{i} \forall i>I$. Since $\mathfrak{S}_{\bar{r}} F^{i}$ converges to $\mathfrak{S}_{\bar{r}} \mathfrak{F}_{\bar{r}}$, it follows that $x \in \mathfrak{S}_{\bar{r}} \mathfrak{F}_{\bar{r}}$. So, $x \in \mathfrak{S}_{\bar{r}} \mathfrak{F}_{\bar{r}} \cap O_{p}^{o}$.

From what has been proved, one may conclude that as $r$ increases, the sets $F_{r}$ 'expand' in the following way: if $\bar{r} \geq r$, then $F_{r}=\mathfrak{S}_{r} \mathfrak{F}_{r} \cap O_{r}^{o}=\mathfrak{S}_{\bar{r}} \mathfrak{F}_{\bar{r}} \cap O_{r}^{o}=\mathfrak{S}_{\bar{r}} \mathfrak{F}_{\bar{r}} \cap O_{\bar{r}}^{o} \cap O_{r}^{o}=F_{\bar{r}} \cap O_{r}^{o}$ (i.e., $F_{r}$ is a subset of $F_{\bar{r}}$ containing elements $x$ such that $\varrho_{X}(\theta, x)<r$.) Define now the set $F \doteq \bigcup_{r>r_{0}} F_{r}$ and show it is closed. Note that for every $r, F \cap O_{r}^{o}=F_{r}$ holds and the set $F_{r} \cup \overline{O_{r}^{o}}=\mathfrak{S}_{r} F$ is closed. Consider a sequence $\left\{y_{i}\right\}_{i=1}^{\infty} \subset F$ convergent to $y$. This sequence is bounded, so starting with some index $i$, the inclusions $y_{i} \in O_{p}^{o}, y_{i} \in \mathfrak{S}_{p} F$, where $p=$ $\varrho_{X}(\theta, y)+1$, hold. Thus, since $\mathfrak{S}_{p} F$ is closed, it follows that $y \in \mathfrak{S}_{p} F$; on the other hand, $y \notin \overline{O_{r}^{o}}$. So, $y \in F_{p} \subset F$ and $F$ is closed.

Finally, show that the sequence $\left\{F^{i}\right\}_{i=1}^{\infty}$ converges to the set $F$ with respect to the metric $\rho_{\text {cl }}$. For an arbitrary $\varepsilon>0$, put $\bar{r}(\varepsilon)=2 / \varepsilon$. Since the sequence $\left\{\mathfrak{S}_{\bar{r}(\varepsilon)} F^{i}\right\}_{i=1}^{\infty}$ converges (in the Hausdorff metric) to the set $\mathfrak{S}_{\bar{r}(\varepsilon)} \mathfrak{F}_{\bar{r}(\varepsilon)}=\mathfrak{S}_{\bar{r}(\varepsilon)} F$, for every $i$, starting with some $I$, the following inequalities hold:

$$
\operatorname{dist}\left(\mathfrak{S}_{\bar{r}(\varepsilon)} F^{i}, \mathfrak{S}_{\bar{r}(\varepsilon)} F\right)<\varepsilon / 2, \quad \rho^{o}\left(F^{i}, F\right)<\varepsilon / 2
$$

According to Lemma 1, the function $r \mapsto \operatorname{dist}\left(\mathfrak{S}_{r} F^{i}, \mathfrak{S}_{r} F\right)$ is non-decreasing. Hence, from (12), it follows that $\operatorname{dist}\left(\mathfrak{S}_{r} F^{i}, \mathfrak{S}_{r} F\right)<\varepsilon / 2$ for every $r \leq \bar{r}(\varepsilon)$. Next, for $r>\bar{r}(\varepsilon)$, one has $1 / r<\varepsilon / 2$. Thus, $\rho^{\mathfrak{S}}\left(F^{i}, F\right) \leq \varepsilon / 2$ for all $i>I$, and therefore $\rho_{\mathrm{cl}}\left(F^{i}, F\right)<\varepsilon$, i.e., $\lim _{i \rightarrow \infty} \rho_{\mathrm{cl}}\left(F^{i}, F\right)=0$.

Remark 1 The function $\rho^{\mathfrak{S}}: \operatorname{clos}(X) \times \operatorname{clos}(X) \rightarrow \mathbb{R}_{+}$, given by (4), also defines a metric in the space $\operatorname{clos}(X)$, but for a complete metric space $X$, the space $\left(\operatorname{clos}(X), \rho^{\mathfrak{S}}\right)$, unlike 
( $\operatorname{clos}(X), \rho_{\mathrm{cl}}$ ), may not be complete. For example, the sequence of the sets $F^{i} \doteq\{i\}$ (each consisting of a natural number) in the space $\left(\operatorname{clos}(\mathbb{R}), \rho^{\mathfrak{S}}\right)$ is fundamental [4]:

$$
\forall j>i \quad \rho^{\mathfrak{S}}(\{i\},\{j\})=\frac{8}{\sqrt{i^{2}+4}+i},
$$

yet there is no nonempty set $F \in \operatorname{clos}(\mathbb{R})$ such that

$$
\rho^{\mathfrak{S}}\left(F^{i}, F\right) \rightarrow 0 .
$$

Let us show this. If such a set $F \subset \mathbb{R}$ does exist, then taking any of its points $x_{0}$, one gets the following estimates: for every $i \geq\left|x_{0}\right|+1$ and each $r \in\left[\left|x_{0}\right|, i\right]$,

$$
\operatorname{dist}\left(\mathfrak{S}_{r} F^{i}, \mathfrak{S}_{r} F\right) \geq r-\left|x_{0}\right|
$$

Thus, $\rho^{\mathfrak{S}}\left(F^{i}, F\right) \geq 1 / \bar{r}$, where $\bar{r}$ is a solution of the equation $1 / r=r-\left|x_{0}\right|$. So, the inequality

$$
\rho^{\mathfrak{S}}\left(F^{i}, F\right) \geq \frac{2}{\left|x_{0}\right|+\sqrt{x_{0}^{2}+4}}
$$

holds, which contradicts relation (13).

The Hausdorff distance between sets $A, B \in \operatorname{clos}(X)$ has a simple geometric interpretation:

$$
\operatorname{dist}(A, B)=\inf \left\{\varepsilon: \varepsilon>0, A \subset O_{\varepsilon}^{o}(B), B \subset O_{\varepsilon}^{o}(A)\right\}
$$

Regarding the metric $\rho_{\mathrm{cl}}$, the analogue of the $\varepsilon$-neighborhood of a set $A$ is given by

$$
O_{\varepsilon}^{o}(A, \mathrm{cl}) \doteq \bigcup_{x_{0} \in \mathfrak{A}_{\varepsilon}}\left\{x \in X: \varrho_{X}\left(x, x_{0}\right)<\varepsilon\right\}, \quad \mathfrak{A}_{\varepsilon}=A \cup \overline{O_{1 / \varepsilon}^{o}}
$$

(i.e., by the $\varepsilon$-neighborhood of the set $\mathfrak{A}_{\varepsilon}$ ). This means that the 'main part' $\rho^{\mathfrak{G}}$ of the distance $\rho_{\mathrm{cl}}$ can be defined by the equality

$$
\rho^{\mathfrak{S}}(A, B)=\inf \left\{\varepsilon: \varepsilon>0, A \subset O_{\varepsilon}^{o}(B, \mathrm{cl}), B \subset O_{\varepsilon}^{o}(A, \mathrm{cl})\right\} .
$$

Let us now give a criterion for a sequence of closed sets to be convergent with respect to the metric $\rho_{\mathrm{cl}}$.

Lemma 2 Given $F, F_{i} \in \operatorname{clos}(X), i=1,2, \ldots$, the convergence $\rho_{\mathrm{cl}}\left(F_{i}, F\right) \rightarrow 0$ implies $\varrho(\theta$, $\left.F^{i}\right) \rightarrow \varrho(\theta, F)$ and $\operatorname{dist}\left(\mathfrak{S}_{r} F^{i}, \mathfrak{S}_{r} F\right) \rightarrow 0$ for any $r>0$.

Conversely, let $\left\{F^{i}\right\}_{i=1}^{\infty} \subset \operatorname{clos}(X)$. If there exist $\varrho_{0} \doteq \lim _{i \rightarrow \infty} \varrho\left(\theta, F^{i}\right)$ and $r_{0} \geq 0$ such that for every $r>r_{0}$, the sequence $\left\{\mathfrak{S}_{r} F^{i}\right\}_{i=1}^{\infty}$ converges in the metric dist to some set $F_{r} \in \operatorname{clos}(X)$, then for any $r, \bar{r}$ such that $\bar{r}>r>r_{0}, F_{\bar{r}} \cap O_{\bar{r}}^{o} \supset F_{r} \cap O_{r}^{o}$ holds, and in the space $\operatorname{clos}(X)$ the sequence $\left\{F^{i}\right\}_{i=1}^{\infty}$ converges (with respect to $\rho_{\mathrm{cl}}$ ) to the set $F \doteq \bigcup_{r>r_{0}}\left(F_{r} \cap O_{r}^{o}\right) \in \operatorname{clos}(X)$; moreover, $\varrho(\theta, F)=\varrho_{0}$. 
We omit the proof of this statement since it repeats that of the corresponding result for the finite dimensional case [4].

We conclude this section by proving a theorem on the connection between convergence in the metric $\rho_{\mathrm{cl}}$ and convergence in the metric dist. Note that if a sequence $\left\{F^{i}\right\}_{i=1}^{\infty} \subset$ $\operatorname{clos}(X)$ converges in the Hausdorff metric, then by statement (2) of Lemma 1, it converges (to the same limit) in the metric $\rho^{\mathfrak{S}}$; moreover, as it will be shown later, in the metric $\rho_{\mathrm{cl}}$. The converse is not true. For example, in $\mathbb{R}$ the sequence of sets $F^{i} \doteq\{0, i\}, i=1,2, \ldots$, converges in the metric $\rho_{\mathrm{cl}}$ to the set $F \doteq\{0\}$. On the other hand, for any $i$, $j$, one has $\operatorname{dist}\left(F^{i}, F^{j}\right)=|i-j|$, i.e., the sequence $\left\{F^{i}\right\}_{i=1}^{\infty}$ is divergent in the Hausdorff metric.

Theorem 2 Let $F, F^{i} \in \operatorname{clos}(X), i \in \mathbb{N}$. Then the following statements hold:

(1) if $\operatorname{dist}\left(F^{i}, F\right) \rightarrow 0$, then $\rho_{\mathrm{cl}}\left(F^{i}, F\right) \rightarrow 0$;

(2) if $\rho_{\mathrm{cl}}\left(F^{i}, F\right) \rightarrow 0$ and there is a $p>0$ such that $F^{i} \subset O_{p}, i \in \mathbb{N}$, then $F \in O_{p}$ and $\operatorname{dist}\left(F^{i}, F\right) \rightarrow 0$.

Proof (1) Suppose $\operatorname{dist}\left(F^{i}, F\right) \rightarrow 0, i \rightarrow \infty$. Then, for an arbitrary $\varepsilon>0$, there is a number $I=I(\varepsilon)$ such that $\operatorname{dist}\left(F^{i}, F\right)<\varepsilon$ for every $i>I$. So, according to inequality (9), one gets $\left|\varrho\left(\theta, F^{i}\right)-\varrho(\theta, F)\right|<\varepsilon$, and from (6) it follows that the inequality $\operatorname{dist}\left(\mathfrak{S}_{r} F^{i}, \mathfrak{S}_{r} F\right)<\varepsilon$ holds for every $r \geq 0$. Thus, by Lemma $2, \rho_{\mathrm{cl}}\left(F^{i}, F\right) \rightarrow 0$.

(2) Now, let $\rho_{\mathrm{cl}}\left(F^{i}, F\right) \rightarrow 0$. Then from Lemma 2 , it follows that $\operatorname{dist}\left(\mathfrak{S}_{r} F^{i}, \mathfrak{S}_{r} F\right) \rightarrow 0$ for every $r>0$. Next, since for every $i=1,2, \ldots, F^{i} \subset O_{p}$ holds, then $F \subset O_{p}$. Indeed, if this inclusion fails, then there exists an $f \in F$ such that $\delta \doteq \varrho\left(f, O_{p}\right)>0$, so one gets the estimate

$$
\begin{aligned}
\operatorname{dist}\left(\mathfrak{S}_{p+2 \delta} F, \mathfrak{S}_{p+2 \delta} F^{i}\right) & \geq \varrho\left(f, \mathfrak{S}_{p+2 \delta} F^{i}\right)=\varrho\left(f, F^{i} \cup \overline{O_{p+2 \delta}^{o}}\right) \\
& =\min \left\{\varrho\left(f, F^{i}\right), \varrho\left(f, \overline{O_{p+2 \delta}^{o}}\right)\right\} \geq \min \left\{\varrho\left(f, O_{p}\right), \delta\right\}=\delta
\end{aligned}
$$

that contradicts the relation $\operatorname{dist}\left(\mathfrak{S}_{r} F, \mathfrak{S}_{r} F^{i}\right) \rightarrow 0$. Hence $F \subset O_{p}$.

Let $r \geq 3 p$. Since $F^{i} \subset \mathfrak{S}_{r} F^{i}$, for every $i$ one has

$$
d\left(\mathfrak{S}_{r} F^{i}, \mathfrak{S}_{r} F\right) \geq d\left(F^{i}, \mathfrak{S}_{r} F\right)=d\left(F^{i}, F \cup \overline{O_{r}^{o}}\right) .
$$

Next, for every $f \in F^{i}, i \in \mathbb{N}$, we have

$$
\varrho(f, F) \leq 2 p, \quad \varrho\left(f, \overline{O_{r}^{o}}\right) \geq 2 p
$$

and therefore $\varrho(f, F) \leq \varrho\left(f, \overline{O_{r}^{o}}\right)$, i.e., $\varrho(f, F)=\varrho\left(f, F \cup \overline{O_{r}^{o}}\right)$. Thus, $d\left(F^{i}, F \cup \overline{O_{r}^{o}}\right)=d\left(F^{i}, F\right)$ and

$$
d\left(\mathfrak{S}_{r} F^{i}, \mathfrak{S}_{r} F\right) \geq d\left(F^{i}, F\right) .
$$

Similarly, $d\left(\mathfrak{S}_{r} F, \mathfrak{S}_{r} F^{i}\right) \geq d\left(F, F^{i}\right)$. So, it is proved that

$$
\operatorname{dist}\left(\mathfrak{S}_{r} F^{i}, \mathfrak{S}_{r} F\right) \geq \operatorname{dist}\left(F^{i}, F\right)
$$

hence $\operatorname{dist}\left(F^{i}, F\right) \rightarrow 0$. 


\section{Continuous multi-valued maps}

Let $T$ be a topological space. Recall that a map $F: T \rightarrow \operatorname{clos}(X)$ is said to be continuous at a point $t_{0} \in T$ if for any $\varepsilon>0$, one can find a neighborhood $V\left(t_{0}\right)$ of $t_{0}$ such that $\operatorname{dist}\left(F\left(t_{0}\right), F(t)\right)<\varepsilon$ for every $t \in V\left(t_{0}\right)$. This definition corresponds to endowing the space $\operatorname{clos}(X)$ with the Hausdorff metric. In what follows, we call a map satisfying the listed requirements continuous in the Hausdorff metric in order to distinguish it from the one considered in the space $\left(\operatorname{clos}(X), \rho_{\mathrm{cl}}\right)$.

Definition 1 A map $F: T \rightarrow \operatorname{clos}(X)$ is said to be continuous at a point $t_{0} \in T$ in the metric $\rho_{\mathrm{cl}}$ if for any $\varepsilon>0$, there is a neighborhood $V\left(t_{0}\right)$ of $t_{0}$ such that $\rho_{\mathrm{cl}}\left(F\left(t_{0}\right), F(t)\right)<\varepsilon$ for every $t \in V\left(t_{0}\right)$.

Since the space $\operatorname{clbd}(X)$ with the metric $\rho_{\mathrm{cl}}$ is a subspace of $\operatorname{clos}(X)$, one can also apply the given definition to a map $F: T \rightarrow \operatorname{clbd}(X)$, i.e., to a map having closed bounded images.

Following the standard terminology, the map $F$ is called continuous on a set $T_{0} \subseteq T$ in the metric dist or metric $\rho_{\mathrm{cl}}$ if it is continuous in the corresponding metric at every point of $T_{0}$.

\section{Theorem 3}

(1) If $F: T \rightarrow \operatorname{clos}(X)$ is continuous at a point $t_{0} \in T$ in the Hausdorff metric, then it is continuous at $t_{0}$ in the metric $\rho_{\mathrm{cl}}$.

(2) If $F: T \rightarrow \operatorname{clos}(X)$ is continuous at a point $t_{0} \in T$ in the metric $\rho_{\mathrm{cl}}$ and there exist a number $p>0$ and a neighborhood $V\left(t_{0}\right)$ of $t_{0}$ such that $F(t) \subset O_{p}$ for every $t \in V\left(t_{0}\right)$, then $F$ is continuous at $t_{0}$ in the Hausdorff metric.

Proof (1) Suppose $F$ is continuous at some point $t_{0} \in T$ in the Hausdorff metric and let $\varepsilon>0$. Then there is a neighborhood $V\left(t_{0}\right)$ of $t_{0}$ such that $\operatorname{dist}\left(F\left(t_{0}\right), F(t)\right)<\varepsilon / 2$ for every $t \in V\left(t_{0}\right)$. So, for every $t \in V\left(t_{0}\right)$, according to estimate (9), one gets the inequality $\rho^{o}\left(F\left(t_{0}\right), F(t)\right)<\varepsilon / 2$ and, according to (6), the inequality $\rho^{\mathfrak{S}}\left(F\left(t_{0}\right), F(t)\right)<\varepsilon / 2$. Therefore, $\rho_{\mathrm{cl}}\left(F\left(t_{0}\right), F(t)\right)<\varepsilon, t \in V\left(t_{0}\right)$.

(2) Now, let $F: T \rightarrow \operatorname{clos}(X)$ be continuous at a point $t_{0}$ in the metric $\rho_{\mathrm{cl}}$. This means that, for a positive $\varepsilon<\frac{1}{4}\left(\sqrt{p^{2}+8}-p\right)$, there is a neighborhood $U\left(t_{0}\right)$ of $t_{0}$ such that, for every $t \in U\left(t_{0}\right)$, the following inequality holds:

$$
\rho_{\mathrm{cl}}\left(F\left(t_{0}\right), F(t)\right)<\varepsilon
$$

Without loss of generality, assume that $U\left(t_{0}\right) \subset V\left(t_{0}\right)$. From (14), it follows that

$$
\operatorname{dist}\left(\mathfrak{S}_{r} F\left(t_{0}\right), \mathfrak{S}_{r} F(t)\right)<\varepsilon
$$

for every $r<1 / \varepsilon$. Set $r_{0}=p+2 \varepsilon$. Then

$$
r_{0}<p+\frac{\sqrt{p^{2}+8}-p}{2}=\frac{p+\sqrt{p^{2}+8}}{2}=\frac{4}{\sqrt{p^{2}+8}-p}=\frac{1}{\varepsilon},
$$

and hence

$$
\operatorname{dist}\left(\mathfrak{S}_{r_{0}} F\left(t_{0}\right), \mathfrak{S}_{r_{0}} F(t)\right)<\varepsilon
$$


Taking into account the inclusions $F\left(t_{0}\right), F(t) \subset O_{p}$, one gets

$$
\operatorname{dist}\left(F\left(t_{0}\right), \overline{O_{r_{0}}^{o}}\right) \geq 2 \varepsilon, \quad \operatorname{dist}\left(F(t), \overline{O_{r_{0}}^{o}}\right) \geq 2 \varepsilon
$$

From the estimates obtained, it follows that $\operatorname{dist}\left(F\left(t_{0}\right), F(t)\right)<\varepsilon$. In fact,

$$
\varepsilon>d\left(F\left(t_{0}\right) \cup \overline{O_{r_{0}}^{o}}, F(t) \cup \overline{O_{r_{0}}^{o}}\right) \geq d\left(F\left(t_{0}\right), F(t) \cup \overline{O_{r_{0}}^{o}}\right)=d\left(F\left(t_{0}\right), F(t)\right) .
$$

The last equality holds since for every $f \in F\left(t_{0}\right)$, one has

$$
\varrho\left(f, \overline{O_{r_{0}}^{o}}\right) \geq 2 \varepsilon, \quad \varrho\left(f, F(t) \cup \overline{O_{r_{0}}^{o}}\right)<\varepsilon
$$

i.e., $\varrho\left(f, F(t) \cup \overline{O_{r_{0}}^{o}}\right)=\varrho(f, F(t))$. Similarly, $\varepsilon>d\left(F(t), F\left(t_{0}\right)\right)$.

Thus, the map $F$ is continuous at a point $t_{0}$ in the Hausdorff metric.

So, the continuity of $F: T \rightarrow \operatorname{clos}(X)$ in the Hausdorff metric implies its continuity in the metric $\rho_{\mathrm{cl}}$. The following example shows that the converse is not true.

Example 2 Consider the map $F:[1,2] \rightarrow \operatorname{clos}(\mathbb{R})$ given by $F(t) \doteq\left\{3^{n} t, n=0,1,2, \ldots\right\}$. The map $F$ is not only not continuous in the metric dist, but for any $t_{1}, t_{2} \in[1,2], t_{1} \neq t_{2}$, the following holds:

$$
\operatorname{dist}\left(F\left(t_{1}\right), F\left(t_{2}\right)\right)=\sup _{n}\left\{3^{n}\left|t_{1}-t_{2}\right|\right\}=\infty
$$

Show that the map $F$, nevertheless, is continuous with respect to the metric $\rho_{\mathrm{cl}}$ in $\operatorname{clos}(\mathbb{R})$ with $\theta=0$. For arbitrary $t_{1}, t_{2} \in[1,2]$ (assume for definiteness $t_{1}<t_{2}$ ), one has

$$
\rho^{o}\left(F\left(t_{1}\right), F\left(t_{2}\right)\right)=t_{2}-t_{1} .
$$

Evaluate $\rho^{\mathfrak{S}}\left(F\left(t_{1}\right), F\left(t_{2}\right)\right)$. First of all,

$$
\operatorname{dist}\left(\mathfrak{S}_{r} F\left(t_{1}\right), \mathfrak{S}_{r} F\left(t_{2}\right)\right)= \begin{cases}0, & r \in\left[0, t_{1}\right], \\ r-t_{1}, & r \in\left(t_{1}, t_{2}\right], \\ 3^{n}\left(t_{2}-t_{1}\right), & r \in\left(3^{n} t_{2}, 3^{n}\left(t_{2}+2 t_{1}\right)\right], n=0,1,2, \ldots, \\ r-3^{n+1} t_{1}, & r \in\left(3^{n}\left(t_{2}+2 t_{1}\right), 3^{n+1} t_{2}\right], n=0,1,2, \ldots\end{cases}
$$

Then, for every $r \geq 0$,

$$
\operatorname{dist}\left(\mathfrak{S}_{r} F\left(t_{1}\right), \mathfrak{S}_{r} F\left(t_{2}\right)\right) \leq \frac{t_{2}-t_{1}}{t_{2}} r
$$

here the equality is attained at the points $r=0$ and $r=3^{n} t_{2}, n=0,1,2, \ldots$ (see Figure 1). Since $t_{2} \geq 1$, from (16) it follows that

$$
\operatorname{dist}\left(\mathfrak{S}_{r} F\left(t_{1}\right), \mathfrak{S}_{r} F\left(t_{2}\right)\right) \leq\left(t_{2}-t_{1}\right) r \quad \forall r \geq 0 .
$$




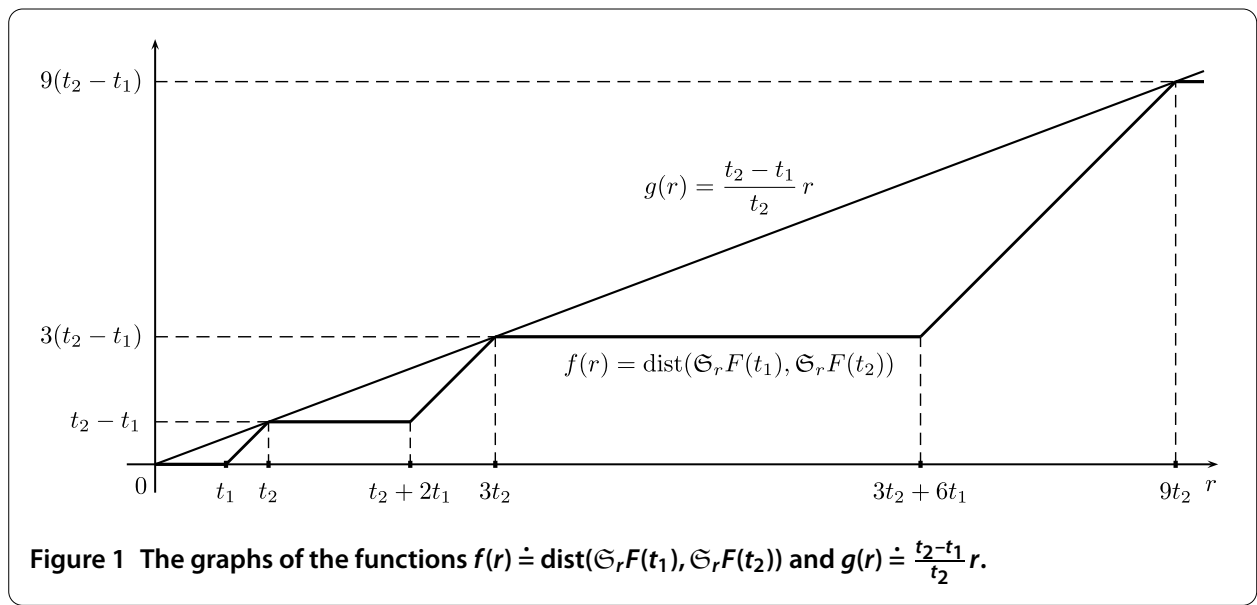

From this inequality, one gets

$$
\rho^{\mathfrak{S}}\left(F\left(t_{1}\right), F\left(t_{2}\right)\right) \leq \frac{1}{\bar{r}},
$$

where $\bar{r}$ satisfies $1 / \bar{r}=\left(t_{2}-t_{1}\right) \bar{r}$. Therefore,

$$
\rho^{\mathfrak{S}}\left(F\left(t_{1}\right), F\left(t_{2}\right)\right) \leq \sqrt{t_{2}-t_{1}}
$$

So, due to relations (15) and (17), $F$ is continuous in the metric $\rho_{\mathrm{cl}}$.

\section{Lipschitz multi-valued maps}

We consider now multi-valued maps defined on a metric space.

Let $\left(\Omega, \varrho_{\Omega}\right)$ be a metric space and $F: \Omega \rightarrow \operatorname{clos}(X)$. Let $q \geq 0$. Recall that the map $F$ is said to be $q$-Lipschitz (or Lipschitz with a constant $q$ ) if for any $\omega_{1}, \omega_{2} \in \Omega$, the following holds:

$$
\operatorname{dist}\left(F\left(\omega_{1}\right), F\left(\omega_{2}\right)\right) \leq q \varrho_{\Omega}\left(\omega_{1}, \omega_{2}\right) .
$$

Naturally, this definition is applicable to a map $F: \Omega \rightarrow \operatorname{clbd}(X)$. In what follows, a map satisfying inequality (18) will be called q-Lipschitz in the Hausdorff metric.

To give the definition of a Lipschitz map in the case when $\operatorname{clos}(X)$ is endowed with the metric $\rho_{\mathrm{cl}}$, note that this metric is given by a pair of functions $\rho^{o}, \rho^{\mathfrak{S}}: \operatorname{clos}(X) \times \operatorname{clos}(X) \rightarrow$ $\mathbb{R}_{+}$defined by (3), (4) (the second of which is a metric itself (see Remark 1$)$ ).

Definition 2 Let $q \geq 0$. A map $F: \Omega \rightarrow \operatorname{clos}(X)$ is said to be $q$-Lipschitz in the metric $\rho^{\mathfrak{S}}$ if for any $\omega_{1}, \omega_{2} \in \Omega$,

$$
\rho^{\mathfrak{S}}\left(F\left(\omega_{1}\right), F\left(\omega_{2}\right)\right) \leq q \varrho_{\Omega}\left(\omega_{1}, \omega_{2}\right)
$$

and $q$-Lipschitz in the metric $\rho_{\mathrm{cl}}$ if for any $\omega_{1}, \omega_{2} \in X$, inequality (19) and the inequality

$$
\rho^{o}\left(F\left(\omega_{1}\right), F\left(\omega_{2}\right)\right) \leq q \varrho_{\Omega}\left(\omega_{1}, \omega_{2}\right)
$$

hold. 
In the case of $\Omega=X$ and $q<1$, a map $F$ satisfying condition (18) is called contracting or $q$ contracting (in the metric dist). Similarly, a map $F: X \rightarrow \operatorname{clos}(X)$ will be called contracting in the metric $\rho^{\mathfrak{S}}$ implying it satisfies inequality (19) with a constant $q<1$, and contracting in the metric $\rho_{\mathrm{cl}}$ if it satisfies inequalities (19), (20).

In what follows, the conditions for a map $F$ to be Lipschitz in the metrics dist, $\rho^{\mathfrak{S}}$, and $\rho_{\mathrm{cl}}$, as well as connections between these properties are derived.

We begin with the criteria of the $q$-Lipschitzness of $F$ in the metrics dist and $\rho_{\mathfrak{S}}$.

\section{Theorem 4}

(1) A map $F: \Omega \rightarrow \operatorname{clos}(X)$ is q-Lipschitz in the metric dist if and only if the map $\mathfrak{S}_{r} F: \Omega \rightarrow \operatorname{clos}(X)$ is q-Lipschitz in the metric dist for each $r \geq 0$.

(2) A map $F: \Omega \rightarrow \operatorname{clos}(X)$ is $q$-Lipschitz in the metric $\rho^{\mathfrak{S}}$ if and only if for every $r_{0}>0$ and any $\omega_{1}, \omega_{2} \in X$ such that $\varrho_{\Omega}\left(\omega_{1}, \omega_{2}\right) \leq\left(q r_{0}\right)^{-1}$, the following holds:

$$
\operatorname{dist}\left(\mathfrak{S}_{r} F\left(\omega_{1}\right), \mathfrak{S}_{r} F\left(\omega_{2}\right)\right) \leq q \varrho_{\Omega}\left(\omega_{1}, \omega_{2}\right) \quad \forall r \leq r_{0}
$$

Proof Statement (1) is a direct consequence of the relations between $\operatorname{dist}\left(F\left(\omega_{1}\right), F\left(\omega_{2}\right)\right)$ and $\operatorname{dist}\left(\mathfrak{S}_{r} F\left(\omega_{1}\right), \mathfrak{S}_{r} F\left(\omega_{2}\right)\right)$ obtained in paragraph (2) of Lemma 1: the necessity follows from estimate (6) and sufficiency from equality (7).

Prove statement (2). Necessity. Let $F$ be $q$-Lipschitz in the metric $\rho^{\mathfrak{S}}$, i.e.,

$$
\sup _{r>0} \min \left\{\operatorname{dist}\left(\mathfrak{S}_{r} F\left(\omega_{1}\right), \mathfrak{S}_{r} F\left(\omega_{2}\right)\right), \frac{1}{r}\right\} \leq q \varrho_{\Omega}\left(\omega_{1}, \omega_{2}\right) \quad \forall \omega_{1}, \omega_{2} \in \Omega
$$

It follows that if $1 / r_{0} \geq q \varrho_{\Omega}\left(\omega_{1}, \omega_{2}\right)$, then

$$
\operatorname{dist}\left(\mathfrak{S}_{r_{0}} F\left(\omega_{1}\right), \mathfrak{S}_{r_{0}} F\left(\omega_{2}\right)\right) \leq q \varrho_{\Omega}\left(\omega_{1}, \omega_{2}\right) .
$$

Therefore, inequality (22) takes place for all $\omega_{1}, \omega_{2}$ such that $\varrho_{\Omega}\left(\omega_{1}, \omega_{2}\right) \leq\left(q r_{0}\right)^{-1}$. Since, according to paragraph (1) of Lemma 1, the function $r \mapsto \operatorname{dist}\left(\mathfrak{S}_{r} F\left(\omega_{1}\right), \mathfrak{S}_{r} F\left(\omega_{2}\right)\right)$ does not decrease for any $\omega_{1}, \omega_{2}$, inequality (22) remains valid for every $r \leq r_{0}$.

Sufficiency. Take arbitrary $\omega_{1}, \omega_{2} \in \Omega, \omega_{1} \neq \omega_{2}$, and determine $\bar{r} \doteq\left(q \varrho_{\Omega}\left(\omega_{1}, \omega_{2}\right)\right)^{-1}$. Then using (21), one gets

$$
\begin{aligned}
\sup _{r>0} \min \left\{\operatorname{dist}\left(\mathfrak{S}_{r} F\left(\omega_{1}\right), \mathfrak{S}_{r} F\left(\omega_{2}\right)\right), \frac{1}{r}\right\} & \leq \max \left\{\sup _{0<r \leq \bar{r}} \operatorname{dist}\left(\mathfrak{S}_{r} F\left(\omega_{1}\right), \mathfrak{S}_{r} F\left(\omega_{2}\right)\right), \sup _{r>\bar{r}}\{1 / r\}\right\} \\
& \leq q \varrho_{\Omega}\left(\omega_{1}, \omega_{2}\right),
\end{aligned}
$$

and thus $\rho^{\mathfrak{S}}\left(F\left(\omega_{1}\right), F\left(\omega_{2}\right)\right) \leq q \varrho_{\Omega}\left(\omega_{1}, \omega_{2}\right)$.

Remark 2 A multi-valued map $G: \Omega \rightarrow \operatorname{clos}(X)$ is said to be $(\varepsilon, q)$-uniformly locally Lipschitz (see, e.g., [8]) if for any $\omega_{1}, \omega_{2} \in \Omega$ such that $\varrho_{\Omega}\left(\omega_{1}, \omega_{2}\right) \leq \varepsilon$, the inequality $\operatorname{dist}\left(G\left(\omega_{1}\right), G\left(\omega_{2}\right)\right) \leq q \varrho_{\Omega}\left(\omega_{1}, \omega_{2}\right)$ holds. So, according to Theorem 4, a map $F: \Omega \rightarrow \operatorname{clos}(X)$ is $q$-Lipschitz in the metric $\rho^{\mathfrak{S}}$ if and only if for any $\varepsilon>0$, the map $\mathfrak{S}_{r} F: \Omega \rightarrow \operatorname{clos}(X)$ is $(\varepsilon, q)$-uniformly locally Lipschitz for all $r \leq(q \varepsilon)^{-1}$. 
The following definition allows to consider an important class of metric spaces often appearing in applications and to which the use of Theorem 4 (and the statements proved further on) is of particular interest.

Definition 3 [8] A metric space $\left(\Omega, \varrho_{\Omega}\right)$ is said to be convex if for any points $\mu, v \in \Omega$, $\mu \neq v$, there exists a point $\omega \in \Omega$ such that

$$
\varrho_{\Omega}(\mu, v)=\varrho_{\Omega}(\mu, \omega)+\varrho_{\Omega}(\omega, v), \quad \omega \neq \mu, \omega \neq v .
$$

We will also need the following statement: if $\left(\Omega, \varrho_{\Omega}\right)$ is complete and convex, then

$$
\forall \mu, v \in \Omega \forall a \in\left(0, \varrho_{\Omega}(\mu, v)\right) \exists \omega \in X \quad \varrho_{\Omega}(\mu, \omega)=a, \quad \varrho_{\Omega}(\omega, v)=\varrho_{\Omega}(\mu, v)-a .
$$

This property of convex metric spaces follows immediately from [9, Theorems 14.1, 15.1].

If $\left(\Omega, \varrho_{\Omega}\right)$ is complete and convex, then paragraph (2) of Theorem 4 can be specified.

Corollary 1 Let $\Omega$ be a complete, convex metric space. Then a map $F: \Omega \rightarrow \operatorname{clos}(X)$ is $q$-Lipschitz in the metric $\rho^{\mathfrak{S}}$ if and only if inequality (21) holds for every $r \geq 0$ and any $\omega_{1}, \omega_{2} \in \Omega$ (i.e., if and only if the map $\mathfrak{S}_{r} F: \Omega \rightarrow \operatorname{clos}(X)$ is q-Lipschitz in the metric dist for every $r \geq 0)$.

Proof Necessity. For $r=0$ and any $\omega_{1}, \omega_{2} \in \Omega$, one has $\mathfrak{S}_{0} F\left(\omega_{1}\right)=\mathfrak{S}_{0} F\left(\omega_{2}\right)=\Omega$, so $\operatorname{dist}\left(\mathfrak{S}_{0} F\left(\omega_{1}\right), \mathfrak{S}_{0} F\left(\omega_{2}\right)\right)=0$ and $(21)$ holds for all $\omega_{1}, \omega_{2}$.

Now, let $r>0$. The map $F$ is $q$-Lipschitz in the metric $\rho^{\mathfrak{S}}$, so according to Theorem 4, for all $\omega_{1}, \omega_{2} \in \Omega$, the inequality $\varrho_{\Omega}\left(\omega_{1}, \omega_{2}\right) \leq(q r)^{-1}$ implies relation (21). Fix some $\omega_{1}$, $\omega_{2}$ satisfying $\varrho_{\Omega}\left(\omega_{1}, \omega_{2}\right)>(q r)^{-1}$ and show that (21) remains valid. By the property (23), there exists a finite set of points $\omega_{1}=\xi_{1}, \xi_{2}, \ldots, \xi_{n}=\omega_{2}$ such that $\varrho_{\Omega}\left(\xi_{i}, \xi_{i+1}\right) \leq(q r)^{-1}, i=$ $1, \ldots, n-1$, and

$$
\varrho_{\Omega}\left(\omega_{1}, \omega_{2}\right)=\varrho_{\Omega}\left(\omega_{1}, \xi_{2}\right)+\varrho_{\Omega}\left(\xi_{2}, \xi_{3}\right)+\cdots+\varrho_{\Omega}\left(\xi_{n-1}, \omega_{2}\right)
$$

So, one gets

$$
\begin{aligned}
& \operatorname{dist}\left(\mathfrak{S}_{r} F\left(\omega_{1}\right), \mathfrak{S}_{r} F\left(\omega_{2}\right)\right) \\
& \quad \leq \operatorname{dist}\left(\mathfrak{S}_{r} F\left(\xi_{1}\right), \mathfrak{S}_{r} F\left(\xi_{2}\right)\right)+\operatorname{dist}\left(\mathfrak{S}_{r} F\left(\xi_{2}\right), \mathfrak{S}_{r} F\left(\xi_{3}\right)\right)+\cdots+\operatorname{dist}\left(\mathfrak{S}_{r} F\left(\xi_{n-1}\right), \mathfrak{S}_{r} F\left(\xi_{n}\right)\right) \\
& \quad \leq \sum_{i=1}^{n-1} q \varrho_{\Omega}\left(\xi_{i}, \xi_{i+1}\right)=q \varrho_{\Omega}\left(\omega_{1}, \omega_{2}\right) .
\end{aligned}
$$

The sufficiency part of the theorem follows directly from Theorem 4.

Note that the hypotheses of completeness and convexity of $\Omega$ in Corollary 1 are crucial. Consider some examples.

Example 3 Let $X=\bigcup_{i \in \mathbb{Z}} X_{i}, \varrho_{X}\left(x_{1}, x_{2}\right)=\left|x_{1}-x_{2}\right|, \theta=0$, where $X_{0}=[-1 ; 1], X_{i}=[2 i ; 2 i+1]$, $X_{-i}=[-2 i-1 ;-2 i], i \in \mathbb{N}$. The space $X$ is complete and, obviously, not convex. Define the 
$\operatorname{map} F: X \rightarrow \operatorname{clos}(X)$ by

$$
F(x) \doteq \begin{cases}\{-3 ; 3\}, & x \in X_{0}, \\ \{-3 ; 2 i+3\}, & x \in X_{i}, i \in \mathbb{N}, \\ \{-2 i-3 ; 3\}, & x \in X_{-i}, i \in \mathbb{N} .\end{cases}
$$

Estimate $\rho^{\mathfrak{S}}\left(F\left(x_{1}\right), F\left(x_{2}\right)\right)$ for arbitrary $x_{1}, x_{2} \in X$.

Evidently, if $x_{1}, x_{2} \in X_{i}, i \in \mathbb{Z}$, then $\rho^{\mathfrak{S}}\left(F\left(x_{1}\right), F\left(x_{2}\right)\right)=0$.

Let $x_{1} \in X_{i_{1}}, x_{2} \in X_{i_{2}}, i_{1}, i_{2}=0,1,2, \ldots$; without loss of generality, assume that $i_{1}<i_{2}$. Then $\operatorname{dist}\left(\mathfrak{S}_{r} F\left(x_{1}\right), \mathfrak{S}_{r} F\left(x_{2}\right)\right)=0$ for $r \leq 2 i_{1}+3$. Next, if $2 i_{1}+3<r \leq 2 i_{1}+4$, then

$$
\mathfrak{S}_{r} F\left(x_{1}\right)=\left(\bigcup_{i \in \mathbb{Z}:|i| \geq i_{1}+1} X_{i}\right) \cup\left\{-3,2 i_{1}+3\right\}, \quad \mathfrak{S}_{r} F\left(x_{2}\right)=\left(\bigcup_{i \in \mathbb{Z}:|i| \geq i_{1}+1} X_{i}\right) \cup\{-3\}
$$

therefore, $\operatorname{dist}\left(\mathfrak{S}_{r} F\left(x_{1}\right), \mathfrak{S}_{r} F\left(x_{2}\right)\right)=1$. Since for the same values of $r, 1 / r<1$ holds, one gets

$$
\rho^{\mathfrak{S}}\left(F\left(x_{1}\right), F\left(x_{2}\right)\right)=\sup _{r>2 i_{1}+3}\left\{\frac{1}{r}\right\}=\frac{1}{2 i_{1}+3}<\frac{1}{3} .
$$

On the other hand, $\varrho_{X}\left(x_{1}, x_{2}\right)=\left|x_{1}-x_{2}\right| \geq 1$. Hence,

$$
\rho^{\mathfrak{S}}\left(F\left(x_{1}\right), F\left(x_{2}\right)\right)<\frac{1}{3} \varrho_{X}\left(x_{1}, x_{2}\right) .
$$

In all the other cases, $i . e$, when $x_{1} \in X_{-i_{1}}, x_{2} \in X_{-i_{2}}, i_{1}, i_{2}=0,1,2, \ldots, i_{1} \neq i_{2}$, or when $x_{1} \in X_{-i_{1}}, x_{2} \in X_{i_{2}}, i_{1}, i_{2}=0,1,2, \ldots, i_{1}^{2}+i_{2}^{2} \neq 0$, doing the analogous calculations one also gets estimation (25).

So, the map $F$ is contracting in the metric $\rho^{\mathfrak{S}}$ with a constant $q=1 / 3$. Moreover, since $\rho^{o}\left(F\left(x_{1}\right), F\left(x_{2}\right)\right)=0$ for any $x_{1}, x_{2}$, the map $F$ is contracting in the metric $\rho_{\mathrm{cl}}$.

However, the map $\mathfrak{S}_{r} F: X \rightarrow \operatorname{clos}(X)$ is not contracting (in the metric dist) for $r>3$. Choosing, for example, $x_{1}=1, x_{2}=2$, for any $r \in(3,4]$, one gets

$$
\begin{aligned}
& \mathfrak{S}_{r} F\left(x_{1}\right)=\{-3,3\} \cup[4,5] \cup[-5,-4] \cup[6,7] \cup[-7,-6] \cup \cdots, \\
& \mathfrak{S}_{r} F\left(x_{2}\right)=\{-3\} \cup[4,5] \cup[-5,-4] \cup[6,7] \cup[-7,-6] \cup \cdots, \\
& \operatorname{dist}\left(\mathfrak{S}_{r} F\left(x_{1}\right), \mathfrak{S}_{r} F\left(x_{2}\right)\right)=1=\varrho_{X}\left(x_{1}, x_{2}\right),
\end{aligned}
$$

i.e., in this case, the Lipschitz constant $q \geq 1$. For the same values $x_{1}=1, x_{2}=2$ and $r=5$, the following hold:

$$
\begin{aligned}
& \mathfrak{S}_{r} F\left(x_{1}\right)=\{-3,3,-5,5\} \cup[6,7] \cup[-7,-6] \cup \cdots, \\
& \mathfrak{S}_{r} F\left(x_{1}\right)=\{-3,-5,5\} \cup[6,7] \cup[-7,-6] \cup \cdots, \\
& \operatorname{dist}\left(\mathfrak{S}_{r} F\left(x_{1}\right), \mathfrak{S}_{r} F\left(x_{2}\right)\right)=2=2 \cdot \varrho_{X}\left(x_{1}, x_{2}\right) .
\end{aligned}
$$

Note that the map considered does not have fixed points in $X$. This means that, for example, in the Nadler theorem, one cannot replace the classical assumption of $F$ be contracting in the metric dist with the one of $F$ be contracting in the metric $\rho^{\mathfrak{S}}$. Nevertheless, 
using the map $\mathfrak{S}_{r} F$ instead of the map $F$ allows to weaken the known conditions of fixed points existence. The corresponding result will be given in the next section.

Example 4 Let $X=\bigcup_{i \in \mathbb{Z}} X_{i}, \varrho_{X}\left(x_{1}, x_{2}\right)=\left|x_{1}-x_{2}\right|, \theta=0$, where $X_{0}=(-1.1 ; 1.1), X_{i}=(2 i ; 2 i+$ 1.1), $X_{-i}=(-2 i-1.1 ;-2 i), i \in \mathbb{N}$. This space is convex, but not complete. Define the map $F: X \rightarrow \operatorname{clos}(X)$ by $(24)$.

Like in Example 3, this map is 1/3-contracting in the metrics $\rho^{\mathfrak{S}}$ and $\rho_{\mathrm{cl}}$ (the corresponding arguments repeat those stated above), but for the map $\mathfrak{S}_{r} F, r=5$, and, for example, $x_{1}=1.05, x_{2}=2.05$, one gets again

$$
\operatorname{dist}\left(\mathfrak{S}_{r} F\left(x_{1}\right), \mathfrak{S}_{r} F\left(x_{2}\right)\right)=2=2 \varrho_{X}\left(x_{1}, x_{2}\right) .
$$

The next theorem establishes a connection between the concepts of Lipschitzness considered in different metrics of the space $\operatorname{clos}(X)$.

\section{Theorem 5}

(1) If a map $F: \Omega \rightarrow \operatorname{clos}(X)$ is $q$-Lipschitz in the Hausdorff metric, then it is $q$-Lipschitz in the metric $\rho_{\mathrm{cl}}$ and all the more in the metric $\rho^{\mathfrak{S}}$.

(2) In the case when $\Omega$ is a complete and convex metric space, the concepts of $q$-Lipschitzness of $F: \Omega \rightarrow \operatorname{clos}(X)$ in the metrics $\rho^{\mathfrak{S}}, \rho_{\mathrm{cl}}$, and dist are equivalent.

We give a simple diagram illustrating this theorem:

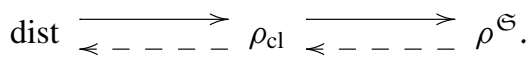

Here, a solid arrow means that the Lipschitzness of $F$ in the first metric implies its Lipschitzness in the second metric; a dashed arrow corresponds to a relation between these properties that takes place only if the metric space $\Omega$ is complete and convex.

Proof (1) The correctness of the implication dist $\rightarrow \rho_{\mathrm{cl}}$ follows from statements (2) and (4) of Lemma 1. Further, by Definition 2, the $q$-Lipschitzness of the map $F$ in the metric $\rho_{\mathrm{cl}}$ implies its $q$-Lipschitzness in the metric $\rho^{\mathfrak{S}}$.

(2) Now, let $\Omega$ be complete, convex and $F: \Omega \rightarrow \operatorname{clos}(X)$ be $q$-Lipschitz in the metric $\rho^{\mathfrak{S}}$. Then, according to Corollary 1 , the map $\mathfrak{S}_{r} F$ is $q$-Lipschitz for every $r \geq 0$ and hence, by statement (1) of Theorem 4, the map $F$ is $q$-Lipschitz in the metric dist. Therefore, $F$ is $q$-Lipschitz in the metric $\rho_{\mathrm{cl}}$ (see paragraph (1) of the theorem).

Let us show that the convexity of a metric space is essential for equivalence of the properties of $q$-Lipschitzness in the metrics dist, $\rho_{\mathrm{cl}}, \rho^{\mathfrak{S}}$.

Note that the maps considered in Examples 3 and 4 are contracting (with a constant $q=1 / 3)$ in the metrics $\rho^{\mathfrak{S}}$ and $\rho_{\mathrm{cl}}$. With respect to the metric dist, they are only Lipschitz with a constant $q>1$. Give an example of a map that is contracting in the metric $\rho_{\mathrm{cl}}$ and not Lipschitz in the metric dist.

Example 5 On the set $\mathbb{N}$, define the metric $\varrho_{\mathbb{N}}(n, m)=|n-m|$ and choose $\theta=1$. Consider $F: \mathbb{N} \rightarrow \operatorname{clos}(\mathbb{N})$ given by $F(n) \doteq\left\{1,(n+1)^{2}\right\}$. Let $n, m \in \mathbb{N}, n<m$. Obviously, 
$\rho^{o}(F(n), F(m))=0$; determine $\operatorname{dist}\left(\mathfrak{S}_{r} F(n), \mathfrak{S}_{r} F(m)\right)$ for different $r \geq 0$. If $r \leq(n+1)^{2}$, then

$$
\begin{aligned}
& \mathfrak{S}_{r} F(n)=\mathfrak{S}_{r} F(m)=\left\{(n+1)^{2},(n+1)^{2}+1,(n+1)^{2}+2, \ldots\right\}, \\
& \operatorname{dist}\left(\mathfrak{S}_{r} F(n), \mathfrak{S}_{r} F(m)\right)=0 .
\end{aligned}
$$

For every $r \in\left((n+1)^{2},(n+1)^{2}+1\right]$, one has

$$
\begin{aligned}
& \mathfrak{S}_{r} F(n)=\left\{(n+1)^{2},(n+1)^{2}+1,(n+1)^{2}+2, \ldots\right\}, \\
& \mathfrak{S}_{r} F(m)=\left\{(n+1)^{2}+1,(n+1)^{2}+2, \ldots\right\} \\
& \operatorname{dist}\left(\mathfrak{S}_{r} F(n), \mathfrak{S}_{r} F(m)\right)=1
\end{aligned}
$$

Since, for the same values of $r, 1 / r<1$ holds, it follows that

$$
\rho^{\mathfrak{S}}(F(n), F(m))=\sup _{r>(n+1)^{2}} \frac{1}{r}=\frac{1}{(n+1)^{2}}<\frac{1}{4}<\frac{1}{4} \varrho_{\mathbb{N}}(n, m) .
$$

So, the map $F$ is $q$-contracting, $q=1 / 4$, in the metric $\rho_{\mathrm{cl}}$. On the other hand,

$$
\operatorname{dist}(F(n), F(m))=(m+1)^{2}-(n+1)^{2}=(m+n+2) \varrho_{\mathbb{N}}(n, m) \quad \forall n, m,
$$

and therefore, there is no constant $q$ for $F$ to be $q$-Lipschitz in the Hausdorff metric.

Next, consider a map that is $q$-Lipschitz in the metric $\rho^{\mathfrak{S}}$ and not $q$-Lipschitz in the metric $\rho_{\mathrm{cl}}$.

Example 6 Let $F: \mathbb{N} \rightarrow \operatorname{clos}(\mathbb{N})$ be given by $F(n) \doteq\left\{(n+1)^{2}\right\}$ (assume $\varrho_{\mathbb{N}}(n, m)=|n-m|$ $\forall n, m \in \mathbb{N}, \theta=1)$. Then, like in Example 5, for any $n, m \in \mathbb{N}(n<m)$, one has

$$
\rho^{\mathfrak{S}}(F(n), F(m))<\frac{1}{4} \varrho_{\mathbb{N}}(n, m),
$$

i.e., inequality (19) is valid with $q=1 / 4$. But

$$
\rho^{o}(F(n), F(m))=(m+1)^{2}-(n+1)^{2}=(m+n+2) \varrho_{\mathbb{N}}(n, m) \quad \forall n, m,
$$

and thus estimate (20) does not hold.

\section{Fixed point theorems}

In this section, we prove a generalization of the Nadler fixed point theorem for a multivalued map $F: X \rightarrow \operatorname{clos}(X)$. The idea of the generalization consists in replacing the image $F(x) \in \operatorname{clos}(X)$ by the set $\mathfrak{S}_{r} F(x) \in \operatorname{clos}(X)$. As it has been mentioned already, this transformation allows to reduce the distance between the images of a map and, in a number of cases, to turn a multi-valued map that is not even continuous into a contracting one. And if it can be proved that a fixed point of the 'new' map $\mathfrak{S}_{r} F: X \rightarrow \operatorname{clos}(X)$ does not belong to $\overline{O_{r}^{o}}$, then this point becomes a fixed one also for the initial map $F$.

Theorem 6 Let $X$ be a complete metric space and $F: X \rightarrow \operatorname{clos}(X)$. Let there exist $r_{0}>0$ and $q \in[0,1)$ such that: 
(1) the map $\mathfrak{S}_{r_{0}} F$ is q-contracting (in the metric dist), i.e.,

$$
\operatorname{dist}\left(\mathfrak{S}_{r_{0}} F\left(x_{1}\right), \mathfrak{S}_{r_{0}} F\left(x_{2}\right)\right) \leq q \varrho_{X}\left(x_{1}, x_{2}\right) \quad \forall x_{1}, x_{2} \in X
$$

(2) there exists $x_{0} \in X$ such that

$$
\varrho_{X}\left(\theta, x_{0}\right)+\frac{\varrho\left(x_{0}, \mathfrak{S}_{r_{0}} F\left(x_{0}\right)\right)}{1-q}<r_{0}
$$

Then there is a point $\bar{x} \in X$ satisfying the inclusion $\bar{x} \in F(\bar{x})$ and the estimate $\varrho_{X}(\theta, \bar{x})<r_{0}$.

Proof From assumption (1), by the Nadler theorem (see $[6,8]$ ), it follows that for every $x_{0} \in X$ and any $\varepsilon>0$, there is a fixed point $\bar{x}$ of the map $\mathfrak{S}_{r_{0}} F$ satisfying the inequality

$$
\varrho_{X}\left(x_{0}, \bar{x}\right)<\frac{\varrho\left(x_{0}, \mathfrak{S}_{r_{0}} F\left(x_{0}\right)\right)+\varepsilon}{1-q} .
$$

Choose $\varepsilon$ so that

$$
\varrho_{X}\left(\theta, x_{0}\right)+\frac{\varrho\left(x_{0}, \mathfrak{S}_{r_{0}} F\left(x_{0}\right)\right)+\varepsilon}{1-q}<r_{0}
$$

Because of inequality (27), such an $\varepsilon$ does exist. Then, from estimates (28), (29), one gets

$$
\varrho_{X}(\theta, \bar{x}) \leq \varrho_{X}\left(\theta, x_{0}\right)+\varrho_{X}\left(x_{0}, \bar{x}\right) \leq \varrho_{X}\left(\theta, x_{0}\right)+\frac{\varrho\left(x_{0}, \mathfrak{S}_{r_{0}} F\left(x_{0}\right)\right)+\varepsilon}{1-q}<r_{0} .
$$

So, $\bar{x} \in \mathfrak{S}_{r_{0}} F(\bar{x})$ and $\bar{x} \notin \overline{O_{r_{0}}^{o}}$, and it follows that $\bar{x}$ is a fixed point of the map $F$.

In the classical theorem of Nadler, if a map $F: X \rightarrow \operatorname{clos}(X)$ is contracting (in the Hausdorff metric) with a constant $q<1 / 2$ and with a fixed point $\bar{x} \in X$ satisfying the condition $F(\bar{x}) \neq\{\bar{x}\}$, then this map has at least one more fixed point different from $\bar{x}$ (see [6]). For maps meeting the requirements of Theorem 6 , the analogous statement may not be true. In fact, it is easy to explain: arguing as in [6, Theorem 2.1.3], take $u_{0} \in \mathfrak{S}_{r_{0}} F(\bar{x}), u_{0} \notin \bar{x}$, as an initial point and, using the iteration procedure, find a fixed point $\bar{u}$ of the map $\mathfrak{S}_{r_{0}} F$. Since $q<1 / 2$, it is easy to show that $\bar{u} \neq \bar{x}$. It may turn out, however, that $\bar{u} \in \overline{O_{r_{0}}^{o}}$ and $\bar{u} \notin F(\bar{u})$, i.e., the point $\bar{u}$ is not a fixed point of the map $F$. This situation does not occur if the element $u_{0} \in F(\bar{x})$ satisfies condition (2) of Theorem 6 . Thus, the following statement holds.

Corollary 2 Let $\mathfrak{S}_{r_{0}} F$ be a contracting map with $q<1 / 2$, and let $\bar{x}$ satisfying $\varrho_{X}(\theta, \bar{x})<r_{0}$ be a fixed point of the map $F$. Then, if there is a point $u_{0} \in F(\bar{x}), u_{0} \neq \bar{x}$, such that

$$
\varrho_{X}\left(\theta, u_{0}\right)+\frac{\varrho_{X}\left(u_{0}, F\left(u_{0}\right)\right)}{1-q}<r_{0}
$$

then $F$ has at least one more fixed point $\bar{u} \neq \bar{x}$. 
Remark 3 An attempt to 'make' the map $F$ a contracting one replacing it by the map $F \cap O_{r_{0}}: X \rightarrow \operatorname{clbd}(X)$ turns out to be far less efficient than using the map $\mathfrak{S}_{r_{0}} F$. This follows from the obvious estimate (see inequality (8))

$$
\begin{aligned}
\operatorname{dist}\left(\mathfrak{S}_{r_{0}} F\left(x_{1}\right), \mathfrak{S}_{r_{0}} F\left(x_{2}\right)\right) & =\operatorname{dist}\left(\left(F\left(x_{1}\right) \cap O_{r_{0}}\right) \cup \overline{O_{r_{0}}^{o}},\left(F\left(x_{2}\right) \cap O_{r_{0}}\right) \cup \overline{O_{r_{0}}^{o}}\right) \\
& \leq \operatorname{dist}\left(F\left(x_{1}\right) \cap O_{r_{0}}, F\left(x_{2}\right) \cap O_{r_{0}}\right) .
\end{aligned}
$$

Example 7 Consider the map $F: \mathbb{R} \rightarrow \operatorname{clos}(\mathbb{R})$ given by $F(x) \doteq\left\{e^{x+i-3}, i \in \mathbb{N}\right\}$ (assume $\varrho_{\mathbb{R}}\left(x_{1}, x_{2}\right)=\left|x_{1}-x_{2}\right|, \theta=0$ ). We show first that this map is not $q$-Lipschitz (for no $q$ ) and, moreover, at any point is neither upper semicontinuous nor lower semicontinuous. More precisely, we prove that for any $x_{1}, x_{2} \in \mathbb{R}$ close enough, $d\left(F\left(x_{1}\right), F\left(x_{2}\right)\right)=\infty$, $d\left(F\left(x_{2}\right), F\left(x_{1}\right)\right)=\infty$ hold.

Without loss of generality, assume $x_{1}<x_{2}$. In order to evaluate the deviation

$$
d\left(F\left(x_{1}\right), F\left(x_{2}\right)\right)=\operatorname{supinf}_{j}\left|e^{x_{2}+i-3}-e^{x_{1}+j-3}\right|,
$$

prove that

$$
0<e^{x_{2}+j-3}-e^{x_{1}+j-3}<\left|e^{x_{2}+i-3}-e^{x_{1}+j-3}\right|
$$

for any $i, j \in \mathbb{N}, i \neq j$, and all $x_{1}, x_{2}$ such that

$$
x_{2}-x_{1}<\ln \left(\frac{2 e}{e+1}\right)
$$

Consider two situations: $i<j$ and $i>j$.

Let $i<j$, then $x_{2}+i<x_{1}+\ln \left(\frac{2 e}{e+1}\right)+i<x_{1}+i+1 \leq x_{1}+j$ and hence,

$$
0<\frac{e^{x_{2}+j-3}-e^{x_{1}+j-3}}{e^{x_{1}+j-3}-e^{x_{2}+i-3}}=\frac{e^{x_{2}-x_{1}}-1}{1-e^{x_{2}-x_{1}-(j-i)}} \leq \frac{e^{x_{2}-x_{1}}-1}{1-e^{x_{2}-x_{1}-1}}<\frac{\frac{2 e}{e+1}-1}{1-\frac{2 e}{(e+1) e}}=1 .
$$

So, inequality (31) is correct.

If $i>j$, then $e^{x_{2}+i-3}>e^{x_{2}+j-3}>e^{x_{1}+j-3}$, and inequality (31) holds as well.

Now, calculate the deviation (30) when $x_{1}, x_{2}$ satisfy condition (32). Due to inequality (31), one gets

$$
d\left(F\left(x_{1}\right), F\left(x_{2}\right)\right) \geq \sup _{j}\left(e^{x_{2}+j-3}-e^{x_{1}+j-3}\right)=\left(e^{x_{2}}-e^{x_{1}}\right) \sup _{j} e^{j-3}=\infty .
$$

For evaluating the deviation $d\left(F\left(x_{2}\right), F\left(x_{1}\right)\right)$, it can be shown in the same manner that if $0<x_{1}-x_{2}<\ln \left(\frac{e+1}{2}\right)$, then for any $i, j$, the inequality

$$
0<e^{x_{2}+i-3}-e^{x_{1}+i-3}<\left|e^{x_{2}+i-3}-e^{x_{1}+j-3}\right|
$$

holds and therefore,

$$
d\left(F\left(x_{2}\right), F\left(x_{1}\right)\right)=\operatorname{supinf}_{i}\left|e^{x_{2}+i-3}-e^{x_{1}+j-3}\right| \geq \sup _{i}\left(e^{x_{2}+i-3}-e^{x_{1}+i-3}\right)=\infty .
$$




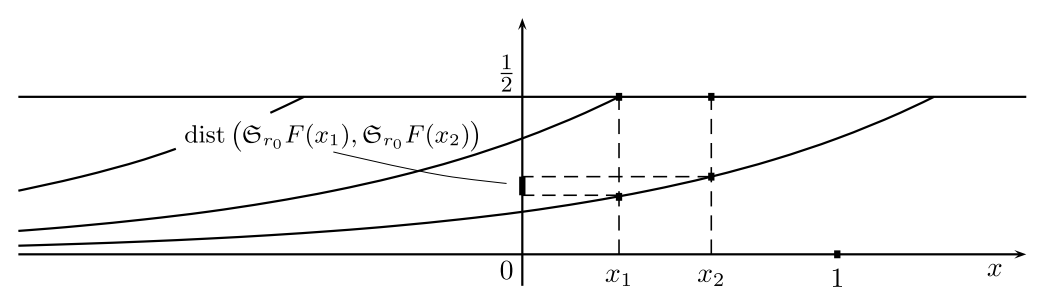

Figure 2 The graph of the map $\mathfrak{S}_{r_{0}} F$.

Despite the fact that $d\left(F\left(x_{1}\right), F\left(x_{2}\right)\right)=d\left(F\left(x_{2}\right), F\left(x_{1}\right)\right)=\infty$ for any close enough $x_{1}, x_{2}$, Theorem 6 can be applied to the map $F$. Put $r_{0}=1 / 2, \theta=0, x_{0}=0$ and prove that relations (26) and (27) are valid. To determine the set $\mathfrak{S}_{r_{0}} F(x)$, we will use equality $\left(2^{\prime}\right)$ since in the space $\mathbb{R}$ definitions (2) and $\left(2^{\prime}\right)$ lead to the same values of $\rho^{\mathfrak{S}}, \rho_{\mathrm{cl}}$ (see the footnote on page 2), but the calculations by means of $\left(2^{\prime}\right)$ are less ponderous.

First of all, note that the map $\mathfrak{S}_{r_{0}} F: \mathbb{R} \rightarrow \operatorname{clbd}(\mathbb{R}), \mathfrak{S}_{r_{0}} F(x)=\left(F(x) \cap O_{r_{0}}^{o}\right) \cup S_{r_{0}}$, has a countable family of continuous selections $f_{i}: \mathbb{R} \rightarrow(0 ; 1 / 2], i \in \mathbb{N}$,

$$
f_{i}(x) \doteq \begin{cases}e^{x+i-3}, & x \in(-\infty ; 3-i-\ln 2] \\ 1 / 2, & x \in(3-i-\ln 2 ;+\infty)\end{cases}
$$

and $\mathfrak{S}_{r_{0}} F(x)=\bigcup_{i}\left\{f_{i}(x)\right\}$ (see Figure 2). Therefore,

$$
\operatorname{dist}\left(\mathfrak{S}_{r_{0}} F\left(x_{1}\right), \mathfrak{S}_{r_{0}} F\left(x_{2}\right)\right) \leq \sup _{i}\left|f_{i}\left(x_{1}\right)-f_{i}\left(x_{2}\right)\right|
$$

For arbitrary $x_{1}, x_{2} \in \mathbb{R}$ and any $i \in \mathbb{N}, f_{i}\left(x_{2}\right)-f_{i}\left(x_{1}\right)=\int_{x_{1}}^{x_{2}} g_{i}(x) d x$ holds, where

$$
g_{i}(x) \doteq \begin{cases}e^{x+i-3}, & x \in(-\infty ; 3-i-\ln 2] \\ 0, & x \in(3-i-\ln 2 ;+\infty) .\end{cases}
$$

Since $0 \leq g_{i}(x) \leq 1 / 2$, the following estimate holds:

$$
\left|f_{i}\left(x_{2}\right)-f_{i}\left(x_{1}\right)\right| \leq \frac{1}{2}\left|x_{2}-x_{1}\right| \quad \forall x_{1}, x_{2},
$$

i.e., the map $\mathfrak{S}_{r_{0}} F$ is contracting with a constant $q=1 / 2$.

Further, taking into account that $x_{0}=\theta=0$, one gets $\varrho\left(0, \mathfrak{S}_{r_{0}} F(0)\right)=\varrho_{\mathbb{R}}\left(0, f_{1}(0)\right)=e^{-2}$,

$$
\varrho_{\mathbb{R}}\left(\theta, x_{0}\right)+\frac{\varrho\left(x_{0}, \mathfrak{S}_{r_{0}} F\left(x_{0}\right)\right)}{1-q}=2 \varrho\left(0, \mathfrak{S}_{r_{0}} F(0)\right)=2 e^{-2}<\frac{1}{2}=r_{0},
$$

so condition (2) of Theorem 6 is also satisfied. Thus, there exists a fixed point $\bar{x}$ of the map $F$ such that $|\bar{x}|<1 / 2$ (it is easy to check that $\bar{x}$ is the minimal solution of the equation $\left.e^{x-2}=x\right)$.

In conclusion, note that in the example considered, replacing the map $F: \mathbb{R} \rightarrow \operatorname{clos}(\mathbb{R})$ by the map $F_{r_{0}} \doteq F \cap O_{r_{0}}: \mathbb{R} \rightarrow \operatorname{clbd}(\mathbb{R})$ does not allow to apply the Nadler theorem. Unlike the contractive map $\mathfrak{S}_{r_{0}} F: \mathbb{R} \rightarrow \operatorname{clbd}(\mathbb{R})$, the map $F_{r_{0}}$ is not defined on $(2-\ln 2 ;+\infty)$ and 


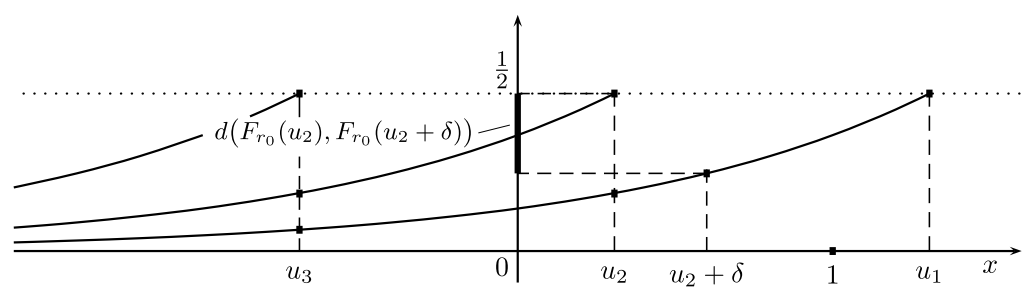

Figure 3 The graph of the map $F_{r_{0}}$.

is not lower semicontinuous at points $u_{i} \doteq 3-i-\ln 2, i \in \mathbb{N}$. Indeed, $F(x) \cap O_{r_{0}}=\varnothing, x>$ $2-\ln 2$; for every $i=2,3, \ldots$ and any $\delta>0$, one has

$$
d\left(F_{r_{0}}\left(u_{i}\right), F_{r_{0}}\left(u_{i}+\delta\right)\right) \geq e^{u_{i}+i-3}-e^{u_{i}+\delta+i-4}=\frac{1}{2}-\frac{e^{\delta-1}}{2},
$$

and hence $d\left(F_{r_{0}}\left(u_{i}\right), F_{r_{0}}\left(u_{i}+\delta\right)\right) \rightarrow \frac{1}{2}-\frac{1}{2 e} \neq 0$ as $\delta \rightarrow 0+0$ (see Figure 3).

\section{Competing interests}

The authors declare that they have no competing interests.

\section{Authors' contributions}

All authors contributed equally and significantly in writing this article. All authors read and approved the final manuscript.

\section{Endnote}

a Note that in the case of linear normed $X$, the values of $\rho^{\mathfrak{S}}$ and $\rho_{\mathrm{cl}}$ calculated by means of (4) and (5) do not change if one defines the operator $\mathfrak{S}_{r}: \operatorname{clos}(X) \rightarrow \operatorname{clos}(X)$ by the equality

$$
\mathfrak{S}_{r} H \doteq\left(H \cap O_{r}^{o}\right) \cup S_{r} \text {. }
$$

This very definition was used in [4] for $X=\mathbb{R}^{n}$. Using (2') for an arbitrary metric space does not seem possible since, first of all, a sphere $S_{r}$ may result in an empty set and as a consequence, the set $\mathfrak{S}_{r} H$ will be empty when $\varrho(\theta, H)>r$.

Received: 27 August 2012 Accepted: 22 December 2012 Published: 10 January 2013

\section{References}

1. Panasenko, EA, Tonkov, EL: Invariant and stably invariant sets for differential inclusions. Proc. Steklov Inst. Math. 262, 194-212 (2008)

2. Panasenko, EA, Tonkov, EL: Extension of E.A. Barbashin's and N.N. Krasovskii's stability theorems to controlled dynamical systems. Proc. Steklov Inst. Math. 268(Suppl. 1), 204-221 (2010)

3. Panasenko, EA, Rodina, LI, Tonkov, EL: The space clcv(Rn) with the Hausdorff-Bebutov metric and differential inclusions. Proc. Steklov Inst. Math. 275(Suppl. 1), 121-136 (2011)

4. Zhukovskiy, ES, Panasenko, EA: On one metric in the space of nonempty closed subsets of Rn. Bull. Udmurt Univ. Math., Mech., Comput. Sci. 1, 15-25 (2012)

5. Panasenko, EA: Dynamical system of translations in the space of multi-valued functions with closed images. Bull. Udmurt Univ. Math., Mech., Comput. Sci. 2, 28-33 (2012)

6. Borisovich, YG, Gel'man, BD, Myshkis, AD, Obukhovskii, W: Introduction to the Theory of Multi-Valued Maps and Differential Inclusions. Akad. Nauk USSR, Moscow (2005) (in Russian)

7. Price, GB: On the completeness of a certain metric space with an application to Blaschke's selection theorem. Bull. Am. Math. Soc. 46(4), 278-280 (1940)

8. Nadler, S Jr.: Multi-valued contraction mappings. Pac. J. Math. 30(2), 475-488 (1969)

9. Blumenthal, LM: Theory and Applications of Distance Geometry. Chelsea, New York (1970) 